TITLE:

\title{
Mechanism for the Formation of Equatorial Superrotation in Forced Shallow-Water Turbulence with Newtonian Cooling
}

\section{$\operatorname{AUTHOR}(\mathrm{S})$ :}

Saito, Izumi; Ishioka, Keiichi

\section{CITATION:}

Saito, Izumi ... [et al]. Mechanism for the Formation of Equatorial Superrotation in Forced Shallow-Water Turbulence with Newtonian Cooling. Journal of the Atmospheric Sciences 2015, 72(4): 1466-1483

ISSUE DATE:

2015-04

URL:

http://hdl.handle.net/2433/203097

RIGHT:

(c) Copyright 2015 American Meteorological Society (AMS). 


\title{
Mechanism for the Formation of Equatorial Superrotation in Forced Shallow-Water Turbulence with Newtonian Cooling
}

\author{
IZUMI SAITO AND KEIICHI ISHIOKA \\ Division of Earth and Planetary Sciences, Graduate School of Science, Kyoto University, Kyoto, Japan
}

(Manuscript received 12 August 2014, in final form 13 November 2014)

\begin{abstract}
Forced shallow-water turbulence on a rotating sphere with Newtonian cooling is examined with the aim of elucidating the mechanism of the robust formation of equatorial superrotation reported by R. K. Scott and L. M. Polvani. It is shown that the Newtonian cooling term distorts the structure of the Hough modes. This distortion can be visualized as either the westward or eastward tilting of the equiphase line with increasing absolute value of latitude; the structural change of the Hough modes leads to the acceleration of the zonalmean flow. A statistical analysis based on a weak-nonlinear theory predicts that stochastically excited Hough modes generate a prograde equatorial jet, the profile of which is quantitatively consistent with that of the ensemble-averaged zonal-mean flow obtained in nonlinear time evolutions. The predicted prograde equatorial jet originates mainly from the acceleration produced by Rossby modes, the equiphase line of which is tilted westward by the Newtonian cooling term. This tilt of the equiphase line of the Hough modes is clarified and a comparison between the acceleration mechanism presented in the present paper and that in other numerical studies in which equatorial superrotation emerges is made.
\end{abstract}

\section{Introduction}

The zonally banded patterns and latitudinally alternating zonal jets are striking features of the atmospheres of Jupiter and Saturn. To explain the origins of these zonal structures, one series of studies following Busse (1983) considers convective motions extending over a deep planetary atmosphere, while another series following Williams (1978) considers quasi-two-dimensional motions within a shallow surface layer of a planetary atmosphere.

In the latter "shallow layer" context, the effect of the differential rotation due to the spherical geometry of the planets plays a crucial role. This effect (the so-called $\beta$ effect) induces a spatial anisotropy in turbulent flows, resulting in the predominance of latitudinally alternating zonal jets. This zonation process was first revealed by Rhines (1975) in freely decaying turbulence on a $\beta$ plane, and subsequently Vallis and Maltrud (1993) examined

Corresponding author address: Izumi Saito, Division of Earth and Planetary Sciences, Graduate School of Science, Kyoto University, Kitashirakawa Oiwake-cho, Sakyo-ku, Kyoto 606-8502, Japan.

E-mail: izumi@kugi.kyoto-u.ac.jp the process in detail, considering a forced-dissipative case and showing that the anisotropic energy cascade in twodimensional wavenumber space leads to the zonation. The numerical time evolution of a two-dimensional turbulent flow on a rotating sphere was first performed in the aforementioned pioneering work by Williams (1978), revealing the spontaneous emergence of strong zonal jets. Note that the numerical model used there was not a full spherical model in that the numerical model imposed symmetry on the flow field. Full spherical numerical experiments were conducted by Yoden and Yamada (1993) and Nozawa and Yoden (1997), in which latitudinally alternating zonal jets were found to emerge from freely evolving turbulence and forced-dissipative turbulence, respectively.

The studies mentioned in the previous paragraph treated two-dimensional nondivergent flows. By including the effect of the horizontal divergence of a flow, which can be represented as a finite Rossby radius of deformation dependent on the latitude in the quasigeostrophic context, numerical time evolutions of turbulence can reproduce other features observed in Jupiter and Saturn. These include vortical motion predominating in the polar region (Okuno and Masuda 2003) and zonal jets having larger amplitudes near the 
equator (Theiss 2004). Dritschel and McIntyre (2008) provided a comprehensive review of previous studies on the zonation process in the quasigeostrophic context and proposed a theory for predicting jet-to-jet spacing based on the potential vorticity staircase paradigm.

The quasigeostrophic approximation, however, is not applicable near the equator. Hence, shallow-water equations on a rotating sphere are more suitable for dealing with the effect of horizontal divergence. Cho and Polvani (1996) conducted numerical experiments on freely decaying shallow-water turbulence on a rotating sphere and showed the predominance of retrograde equatorial jets as well as latitudinally alternating zonal jets at midlatitudes. Hayashi et al. (2007) explored the parameter (the Rossby and Froude numbers) dependence of the flow-pattern formation in detail and showed that the momentum transport by the Rossby waves is the cause of the retrograde equatorial jets.

Retrograde equatorial jets in decaying shallow-water turbulence are rather robust features in rotating spheres, but these do not correspond to the strong prograde equatorial jets (i.e., equatorial superrotation) observed in the atmospheres of Jupiter and Saturn. Kitamura and Ishioka (2007) conducted a large number of ensemble experiments and showed that if the Froude number is relatively large and the Rossby number is not too small, there is a certain probability that a prograde equatorial jet will emerge even from decaying shallow-water turbulence depending on the initial random field. This probability is, however, much less than $50 \%$ and it is not straightforward to relate this prograde equatorial jet to the equatorial superrotation of Jupiter and Saturn. Even with a small-scale random forcing that mimics small convections, the frequency of emergence of a prograde equatorial jet in ensemble numerical experiments is at most $50 \%$ if Rayleigh friction or hypodiffusion is used for large-scale dissipation. Moreover, this value decreases when the model parameters are chosen corresponding to the Jovian atmosphere (Scott and Polvani 2007).

A breakthrough to overcome the robustness of the retrograde equatorial jet (not corresponding to equatorial superrotation of Jupiter and Saturn) in shallowwater turbulence on a rotating sphere was achieved by changing the dissipation process. Scott and Polvani (2008) adopted Newtonian cooling as a more relevant largescale dissipation process for Jupiter and Saturn and obtained robust strong superrotation from forced-dissipative shallow-water turbulence. They suggested that the equatorial super-/subrotation dependence on the dissipation process stems from the fact that the zonal-mean flow acceleration induced by equatorial waves also depends on the dissipation process, as revealed by Andrews and McIntyre (1976) and more recently examined by Warneford and
Dellar (2014, manuscript submitted to J. Fluid Mech.). They found that the zonal-mean flow acceleration induced by equatorial Rossby waves is prograde at the equator if the waves are dissipated by Newtonian cooling, whereas the acceleration is retrograde if the waves are dissipated by Rayleigh friction. They concluded that the dependence of the zonal-mean flow acceleration on the dissipation process is the cause of the predominance of the equatorial superrotation (subrotation) in the Newtonian cooling (Rayleigh friction) experiments conducted by Scott and Polvani (2008). However, since the zonalmean flow acceleration due to each equatorial Rossby wave under Newtonian cooling/Rayleigh friction has its own latitudinal profile and no quantitative comparison has been made between the acceleration produced by linear waves and the growth of equatorial zonal jets in nonlinear numerical experiments, it still remains unclear to what degree the acceleration caused by equatorial Rossby waves contributes to the robust formation of the equatorial superrotation under Newtonian cooling.

The purpose of the present study is to show quantitatively that the zonal-mean flow acceleration due to wave modes distorted by Newtonian cooling triggers the formation of the robust equatorial superrotation reported by Scott and Polvani (2008). The wave modes on which our analysis is based are Hough modes, which are the eigenmodes of the linearized shallow-water equations on a sphere (Longuet-Higgins 1968) rather than the eigenmodes of the linearized shallow-water equations on the equatorial $\beta$ plane (Matsuno 1966), as used in Warneford and Dellar (2014, manuscript submitted to J. Fluid Mech.). After analyzing how each Hough mode distorted by Newtonian cooling causes zonal-mean flow acceleration, we examine whether randomly excited Hough modes can generate a prograde equatorial jet in the early stages of the time evolution of forced shallowwater turbulence on a rotating sphere. To statistically evaluate the net acceleration of zonal-mean flow produced by the randomly excited Hough modes, we partially follow the quasi-linear approach used by Srinivasan and Young (2012).

The remainder of the present paper is organized as follows. In section 2, we describe ensemble experiments of forced shallow-water turbulence with Newtonian cooling and examine the robustness of the formation of the equatorial superrotation. In section 3 we use eigenvalue analysis of the linearized shallow-water equations with Newtonian cooling to show how the Hough modes are distorted. In section 4, we show that these distorted Hough modes induce second-order zonal-mean flow acceleration and statistically evaluate the net acceleration produced by the randomly excited Hough modes. A summary and discussion are presented in section 5 . 


\section{Ensemble experiments}

\section{a. Model description}

The system under consideration is described by shallowwater equations on a sphere of radius $a_{*}$ rotating at an angular frequency $\Omega_{*}$. Here, the asterisk subscript indicates dimensional variables; variables without this subscript are nondimensionalized. Using $a_{*}$ and $2 \pi / \Omega_{*}$ as the length and time scales, respectively, the shallowwater equations can be nondimensionalized as follows:

$$
\begin{aligned}
\frac{\partial \zeta}{\partial t}= & -\left\{\frac{1}{1-\mu^{2}} \frac{\partial}{\partial \lambda}[(4 \pi \mu+\zeta) U]\right. \\
& \left.+\frac{\partial}{\partial \mu}[(4 \pi \mu+\zeta) V]\right\}+d_{\zeta}+F \\
\frac{\partial D}{\partial t}= & -\left\{\frac{1}{1-\mu^{2}} \frac{\partial}{\partial \lambda}[(4 \pi \mu+\zeta) V]-\frac{\partial}{\partial \mu}[(4 \pi \mu+\zeta) U]\right\} \\
& -\nabla^{2}\left[\eta+\frac{U^{2}+V^{2}}{2\left(1-\mu^{2}\right)}\right]+d_{D}, \text { and } \\
\frac{\partial \eta}{\partial t}= & -\left[\frac{1}{1-\mu^{2}} \frac{\partial}{\partial \lambda}(U \eta)+\frac{\partial}{\partial \mu}(V \eta)\right]-\Phi D+d_{\eta},
\end{aligned}
$$

where $t$ is the time; $\lambda$ is the longitude; $\mu=\sin \varphi$ for latitude $\varphi ; \zeta$ is the vorticity; $D$ is the divergence; $\eta$ is the geopotential anomaly from the global-mean value $\Phi$; $(U, V)=(u, v) \cos \varphi$ for eastward and northward velocity components $u$ and $v$, respectively; $F$ and $d_{(\zeta, D, \eta)}$ represent the forcing and dissipation terms, respectively; and $\nabla^{2}$ is the horizontal Laplacian, which is defined as

$$
\nabla^{2}=\frac{1}{1-\mu^{2}} \frac{\partial^{2}}{\partial \lambda^{2}}+\frac{\partial}{\partial \mu}\left[\left(1-\mu^{2}\right) \frac{\partial}{\partial \mu}\right] .
$$

Since the Rossby deformation radius at the poles nondimensionalized by $a_{*}$ is defined as

$$
L_{D}=\frac{\sqrt{\left(\Omega_{*} / 2 \pi\right)^{2} a_{*}^{2} \Phi}}{2 \Omega_{*} a_{*}}=\frac{\sqrt{\Phi}}{4 \pi},
$$

we can write this as $\Phi=16 \pi^{2} L_{D}^{2}$ and use $L_{D}$ as an experimental parameter.

As for the forcing/dissipation terms imposed on the right-hand side of (1)-(3), we adopt the same type of forcing/dissipation as used by Scott and Polvani (2007, 2008). The forcing $F$ is a small-scale white-in-time random process imposed in a narrow spectral band, which is written as

$$
\begin{aligned}
F(\lambda, \mu, t)=2 \operatorname{Re}\left[\sum_{n=n_{f}-\Delta n / 2}^{n_{f}+\Delta n / 2} \sum_{m=1}^{n} \hat{f}_{n}^{m}(t) P_{n}^{m}(\mu) e^{i m \lambda}\right] \quad \text { and } \\
\hat{f}_{n}^{m}=\sqrt{\frac{2 n(n+1) \varepsilon_{0}}{(2 n+1) \Delta n} \xi_{n}^{m}(t),} \\
\xi_{n}^{m}(t)=\frac{e^{i \theta_{n}^{m}(t)}}{\sqrt{\Delta t}}, \quad(m=1,2, \ldots, n) .
\end{aligned}
$$

Here, $\varepsilon_{0}$ is a measure of the energy injection rate; $P_{n}^{m}(\mu)$ is the associated Legendre function, which is normalized as $\int_{-1}^{1}\left[P_{n}^{m}(\mu)\right]^{2} d \mu=2$; and $\theta_{n}^{m}(t)$ is a uniform random number in $[0,2 \pi]$, which is changed at each time step (interval $\Delta t$ ) of the time integration. Note that no forcing is applied to the zonal modes $(m=0)$. This setting is to ensure consistency with the weak-nonlinear and quasilinear models considered in section 4 . We compared the results of the experiments with and without the zonal components of the forcing and confirmed that there are no significant differences between the two. The temporal structure of the forcing is described by the function $\xi_{n}^{m}(t)$, which is a model for a white-noise process with unit variance and, if $\Delta t$ is infinitesimally small, satisfies

$$
\left\langle\xi_{n}^{m}(t) \xi_{n}^{m}\left(t^{\prime}\right)^{*}\right\rangle=\delta\left(t-t^{\prime}\right)
$$

where $(\cdot)^{*}$ indicates the complex conjugate, the angle brackets indicate the ensemble average, and $\delta\left(t-t^{\prime}\right)$ is the Dirac delta function. We set the middle wavenumber of the forcing $n_{f}=42$ and the width of the spectral band $\Delta n=4$. The settings for $\Delta t$ and $\varepsilon_{0}$ are described later in this section. The dissipation terms are set as follows:

$$
d_{\zeta}=-\nu \nabla^{8} \zeta, \quad d_{D}=-\nu \nabla^{8} D, \quad d_{\eta}=-\nu \nabla^{8} \eta-\frac{1}{\tau_{\mathrm{rad}}} \eta .
$$

That is, each dependent variable is dissipated at small scales by the hyperviscosity term with $\nu$, and the Newtonian cooling effect is imposed only on the geopotential anomaly $\eta$. Here, we set $\tau_{\text {rad }}=0.25 L_{D}^{-2}$, following Scott and Polvani (2008), and the hyperviscosity coefficient as $\nu=10 /\left[N_{T}\left(N_{T}+1\right)\right]^{4}$, where $N_{T}$ is the truncation wavenumber of the spectral method (described below).

We compute the time evolution of (1)-(3) using the spherical spectral method, expanding the dependent variables with the spherical harmonics truncated in a triangular manner with $N_{T}=170$. To calculate the nonlinear terms, we use the standard transform method with an alias-free grid of 512 (zonal) $\times 256$ (meridional). We use the classical fourth-order Runge-Kutta scheme 
(a)

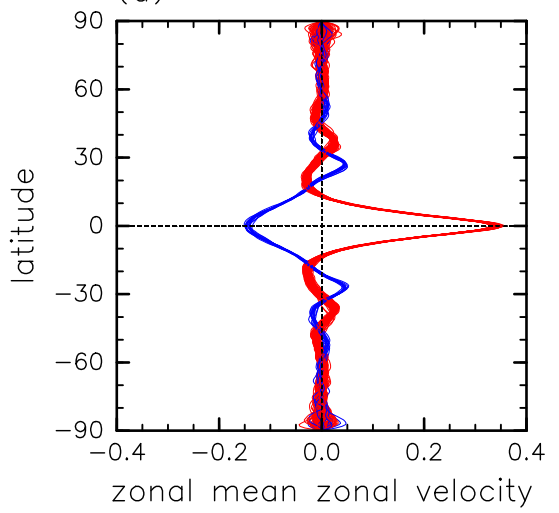

(d)

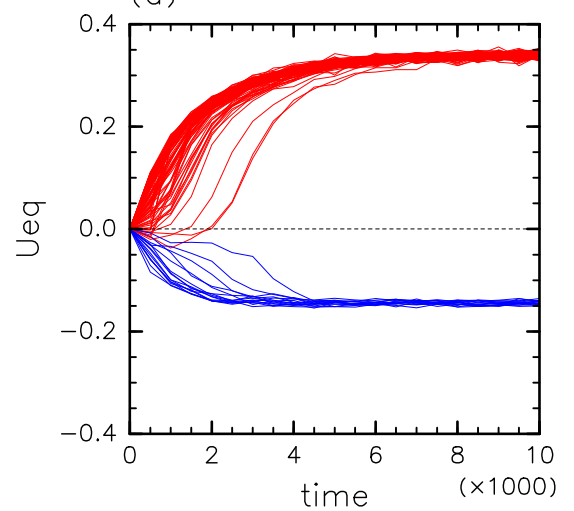

(b)

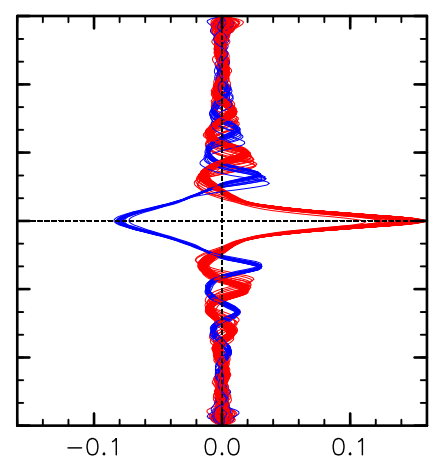

(e)

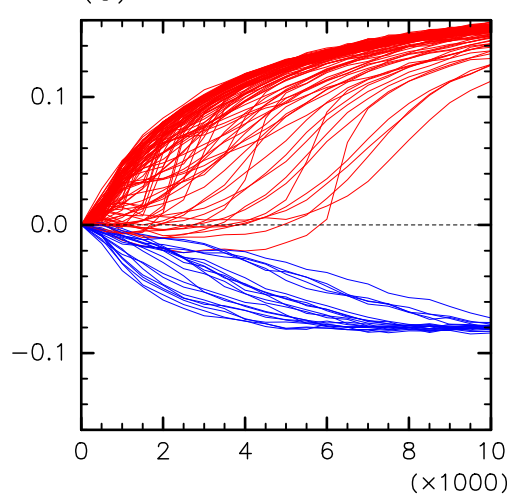

(c)

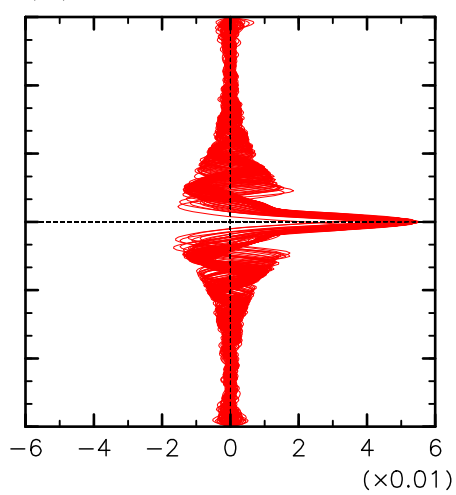

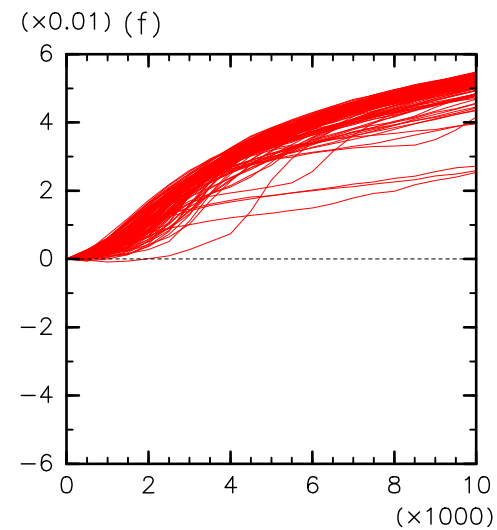

FIG. 1. (a)-(c) Latitudinal profiles of zonal-mean zonal velocity for 120 ensemble members at the end of the time evolution $(t=10000)$ for (a) E6, (b) E7, and (c) E8 experiments. (d)-(f) Time evolutions of the zonal-mean zonal velocity at the equator for 120 ensemble members for (d) E6, (e) E7, and (f) E8 experiments. The red and blue curves indicate the ensemble members with positive and negative equatorial zonal-mean zonal velocities at the end of the time evolution, respectively. The number of red curves is 105 for E6, 99 for E7, and 120 for E8 experiments.

for the time integration with a time increment of $\Delta t=$ 0.0125 . Note that there are some differences between the settings used in the present work and those of Scott and Polvani (2007, 2008), in which second-order leapfrog schemes with Robert-Asselin time filters were adopted for the time integration. The truncation wavenumber used by Scott and Polvani (2007) was the same as that used in the present study, whereas Scott and Polvani (2008) conducted higher-resolution experiments with $N_{T}=682$.

With the above settings, we have two control parameters: the nondimensionalized Rossby deformation radius $L_{D}$ and the measure of the energy injection rate $\varepsilon_{0}$. We set $\varepsilon_{0}=6.4 \times 10^{-E}$ where $E$ is chosen to be 6,7 , or 8 . These are referred to as the "E6," "E7," and "E8" experiments, respectively. We conduct ensemble numerical experiments for 120 members for E6-E8. Scott and Polvani (2008) set $\varepsilon_{0}=5.0 \times 10^{-7}$, so in our work, the setting of $\varepsilon_{0}$ in our E7 experiments provides the closest reproduction of their experiment. For the parameter
$L_{D}$, we use $L_{D}=0.1$, although Scott and Polvani (2008) used three values of $L_{D}: 1.0,0.1$, and 0.025 , among which they considered 0.025 as a typical value for Jupiter and Saturn. One reason why we only use $L_{D}=0.1$ is that, in the case of $L_{D}=1.0$, the maximum phase speed of gravity modes becomes very fast, meaning $\Delta t$ must be very small to yield accurate results, which makes it time consuming to conduct many ensemble experiments. The other reason is that, in the case of $L_{D}=0.025$, the hyperviscosity term becomes comparable to the Newtonian cooling term around the forcing wavenumbers, which may make it difficult to examine the influence of Newtonian cooling on the zonal flow acceleration (this point is discussed in section 5).

\section{b. Results}

While most of the ensemble members yield equatorial superrotation, some yield equatorial subrotation. Figure $1 b$ shows the meridional profiles of the zonal-mean zonal velocities at the end of the time integrations (at $t=10000$ ) 
for the E7 experiments, in which the value of $\varepsilon_{0}$ is closest to that used by Scott and Polvani (2008) as described in the previous subsection. Among the 120 members, 99 members exhibit a strong prograde jet at the equator and latitudinally alternating weak zonal jets at midlatitudes (Fig. 1b, red curves). The meridional profiles of the zonal-mean zonal velocities for these 99 members are very similar to the profile for $L_{D}=0.1$ in Fig. 1 of Scott and Polvani (2008). On the other hand, the other 21 members exhibit a strong retrograde jet at the equator (Fig. 1b, blue curves). The emergence of equatorial retrograde jets seems in discord with the results of Scott and Polvani (2008) because they obtained only equatorial superrotation when Newtonian cooling was the main dissipation. We discuss possible causes of this difference in section 5. For the E8 experiments, where the measure of $\varepsilon_{0}$ is 10 times smaller than that used in the E7 experiments, every member exhibits a prograde jet at the equator (Fig. 1c). We also conducted the same ensemble experiments but with an additional, smaller value of $\varepsilon_{0}$ and confirmed that every member yields a prograde equatorial jet (not shown). On the other hand, for the E6 experiments, where the value of $\varepsilon_{0}$ is 10 times larger than that used in E7 experiments, 15 members exhibit a retrograde jet at the equator (Fig. 1a). There may be a significant difference in the frequency of emergence of prograde equatorial jets between the E6 and E7 experiments.

The time evolutions of the equatorial jets in the ensemble experiments are almost monotonic; that is, in most cases, the equatorial jet grows monotonically, maintaining an either prograde or retrograde direction from the initial stages of its growth. Figures $1 d-f$ show the time evolutions of the zonal-mean zonal velocities at the equator for each ensemble member in E6-E8 experiments, respectively. It can be seen, especially for E6 and E8 (Figs. 1d and 1f, respectively), that in most ensemble members, the equatorial jet has become either prograde or retrograde by $t=1000$, and does not change in direction, but increases in speed monotonically after that. In the E7 experiment, the direction of the equatorial jet changes after $t=1000$ for some ensemble members (Fig. 1e). However, for as many as 88 ensemble members, the jet is prograde by $t=1000$. Note that each time evolution in the E8 and E9 experiments does not reach a statistically steady state by $t=10000$ when the speed of the equatorial jet is still increasing. This is because the dissipation effect of Newtonian cooling on the potential vorticity field is very weak near the equator, and it takes a long time to reach a statistically steady state as discussed by Scott and Polvani (2007).

Although the experimental results shown in this section seem to be in discord with those of Scott and Polvani (2008), in that there emerge retrograde equatorial jets in our experiments, more than $80 \%$ of the ensemble members exhibit equatorial superrotation. Considering that when Rayleigh friction was used as the main dissipation process, the frequency of emergence of a prograde equatorial jet was at most $50 \%$ in the ensemble numerical experiments conducted by Scott and Polvani (2007), it is clear that using Newtonian cooling instead of Rayleigh friction significantly increases this value and that there must be some acceleration mechanism that favors equatorial superrotation. We can make two deductions about this mechanism from our experimental results. First, this acceleration mechanism acts from the beginning of the time evolution and plays a crucial role, particularly in the initial stages of the growth of the equatorial jet, because in most of the ensemble members the equatorial jet does not change direction after $t=1000$, implying that its direction is determined at an early stage. Second, it may be possible that this acceleration mechanism can be described in a weak-nonlinear framework because the emergence of equatorial superrotation is more predominant for forcings of smaller amplitude (especially in E8 experiments).

In the following sections, we focus on the time evolution until $t=1000$ and investigate the acceleration mechanism of the prograde equatorial jet during this early stage.

\section{Structural change of the Hough modes due to Newtonian cooling}

In this section, we examine the effect of Newtonian cooling on the structure of Hough modes. Hough modes are the eigenmodes of the linearized shallow-water equations on a rotating sphere (Longuet-Higgins 1968). If the considered dissipation process is expressed as a linear term of the dependent variable, it is easy to incorporate the dissipation effect into the eigenvalue analysis and obtain the distorted Hough modes.

Pioneering work in this line of study was conducted by Yamagata and Philander (1985). They examined how such linear dissipation terms distort equatorial wave modes, which are the eigenmodes of the linearized shallow-water equations on the equatorial $\beta$ plane, and they showed that the dissipation terms cause tilting of the equiphase lines of each equatorial wave mode. In Yamagata and Philander (1985), there is a very important equation [their (A.6)] that describes the tilting of the equiphase line of the $v$ field and $u$ field for the mixed Rossby-gravity (MRG) mode and the Kelvin mode, respectively. If the coefficient of Rayleigh friction $\alpha$ (here, we have changed the letter denoting the coefficient) and the coefficient of Newtonian cooling $\gamma$ are sufficiently small, then the equation can be approximately written as follows: 


$$
x=\frac{1}{4} \frac{(\gamma-\alpha)}{k \omega_{r}} y^{2}+\text { const } .
$$

Here, $x$ is the eastward coordinate, $y$ is the northward coordinate (the origin is at the equator), $k$ is the zonal wavenumber, and $\omega_{r}$ is the real part of the frequency of the eigenmode. Each variable is nondimensionalized using the $\beta$ parameter and the mean value of the geopotential field. If we consider Newtonian cooling onlythat is, $\gamma>0$ and $\alpha=0$ - then (8) indicates the following:

1) The equiphase line for a westward-propagating mode $\left(\omega_{r}<0\right)$ tilts westward as the absolute value of latitude increases, whereas that for an eastwardpropagating mode tilts eastward.

2) If the zonal wavenumber of the mode is fixed, then the tilt decreases as the frequency $\left|\omega_{r}\right|$ of the mode increases.

Note that (8) and statements 1 and 2 hold exactly only for the $v$ field and $u$ field of the MRG mode and the Kelvin mode of equatorial waves, respectively. Within our results, however, statements 1 and 2 seem to also hold for other dependent variables and Hough modes, including not only the MRG and Kelvin modes but also Rossby and gravity modes individually as shown below. In the following, we present the results of the eigenvalue analysis into which Newtonian cooling was incorporated, while keeping statements 1 and 2 in mind.

We perform eigenvalue analysis of the linearized version of (1)-(3) around the stationary solution $(\zeta=D=\eta \equiv 0)$, omitting the forcing term in (1) and assuming that each dependent variable has the form of $\operatorname{Re}\left[\hat{A}(\mu) e^{i(m \lambda-\omega t)}\right]$. To solve the eigenvalue problem numerically, it is discretized using the same spherical-harmonics expansion as used in the nonlinear time evolution. The value of the parameter $L_{D}$ is also set to the same value (0.1) as used in the nonlinear time evolutions in section 2 . The geopotential anomaly fields of the distorted Hough modes computed as eigenmodes for the zonal wavenumber $m=1$ are shown in Fig. 2. To quantify the tilt of a equiphase line later, the phase of each mode is set so that one of the zero contours passes through the point $(\lambda, \varphi)=\left(0^{\circ}, 90^{\circ}-\varepsilon\right)$, where $\varepsilon$ is infinitesimal. As predicted by statement 1 , it is clearly seen in Fig. 2a that the equiphase line of the Kelvin mode tilts eastward as the absolute value of latitude increases, since the geopotential anomaly field of the Kelvin mode is in phase with the $u$ field. In a neighbor of $(\lambda, \varphi)=\left(0^{\circ}, 0^{\circ}\right)$ in Fig. 2a, one of the zero contours crosses the equator at $(\lambda, \varphi)=\left(\Delta \lambda, 0^{\circ}\right)$, where $\Delta \lambda=-7.6^{\circ}$ in this case. In the following, we use $\Delta \lambda$ as a quantitative measure of the tilt of an equiphase line; a positive (negative) $\Delta \lambda$ corresponds to a westward (eastward) tilt and a larger $\Delta \lambda$ corresponds to a larger tilt. Similarly, in Figs. $2 b$ and $2 c$, it is shown that the equiphase line slightly tilts westward (eastward) as the distance from the equator increases for westward (eastward) propagating MRG modes, although the geopotential anomaly field is not completely in phase with the $v$ field in each hemisphere for these modes $(\Delta \lambda=$ $+2.85^{\circ}$ and $-2.03^{\circ}$ in Figs. $2 \mathrm{~b}$ and $2 \mathrm{c}$, respectively). The tilt of the equiphase line is also in accordance with statement 1 for the gravity and Rossby modes. Each of the eastward-propagating gravity modes shown in Figs. $2 \mathrm{~d}-\mathrm{f}$ has a very slightly eastward-tilting equiphase line $(\Delta \lambda=$ $-0.89^{\circ},-0.49^{\circ}$ and $-0.25^{\circ}$ in Figs. $2 d-f$, respectively). The tilt can also be clearly seen for the Rossby modes shown in Figs. $2 \mathrm{~g}-\mathrm{l}$, for which the equiphase line tilts westward, corresponding to the westward propagation of the Rossby modes. The difference in the extent of the tilt between the gravity and Rossby modes can be understood from statement 2, although it is not directly applicable to these modes as noted above. That is, Rossby modes exhibit larger tilts than gravity modes because of smaller values of $\left|\omega_{r}\right|$. Furthermore, in Figs. $2 \mathrm{~g}-\mathrm{i}$, it is clear that a mode with a smaller associated value of $\left|\omega_{r}\right|$ has a larger tilt among symmetric Rossby modes. Here, we modify the definition of $\Delta \lambda$ as follows: $\Delta \lambda$ is the increment of longitude as one of the zero contours is tracked from $(\lambda, \varphi)=\left(0^{\circ}, 90^{\circ}-\varepsilon\right)$ to the equator. Then, $\Delta \lambda=20^{\circ}, 213^{\circ}$, and $385^{\circ}$ in Figs. $2 \mathrm{~g}-\mathrm{i}$, respectively. For antisymmetric Rossby modes (Figs. 2j-1), the similar relationship between the tilt and $\left|\omega_{r}\right|$ holds. Here, we use the $\Delta \lambda$ modified above except that the zero contour is tracked up to $\varphi=5^{\circ}$ in order to avoid a large phase jump near the equator. Then, $\Delta \lambda=37^{\circ}, 225^{\circ}$, and $391^{\circ}$ in Figs. $2 j-1$, respectively. Although the above definitions of $\Delta \lambda$ may seem a little ad hoc, $\Delta \lambda$ is actually a good measure of the average tilt. In each of Figs. 2g-l, a long-dashed straight line whose slope is $\Delta \lambda / \Delta \varphi$ is drawn, where $\Delta \varphi=90^{\circ}$ and $85^{\circ}$ for symmetric and antisymmetric Rossby modes, respectively. It is clearly seen that the slope of each straight line corresponds well to the average tilt of the equiphase line.

We examined all the distorted Hough modes (including many other modes that are not shown in Fig. 2) calculated in the discretized eigenvalue analysis and qualitatively confirmed that statements 1 and 2 can be regarded as almost general rules in each category of Hough modes at least.

\section{Acceleration of zonal-mean flow due to Hough modes distorted by Newtonian cooling}

In the previous section, it was shown that Newtonian cooling distorts the structure of Hough modes, especially that of Rossby modes. In this section, we investigate the acceleration of zonal-mean flows induced by these distorted Hough modes through a weak-nonlinear analysis. 
(a) $\omega=1.29+\mathrm{i}(-0.021)$

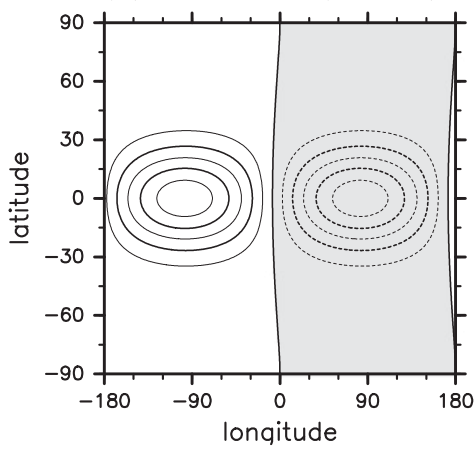

(d) $\omega=9.01+\mathrm{i}(-0.011)$

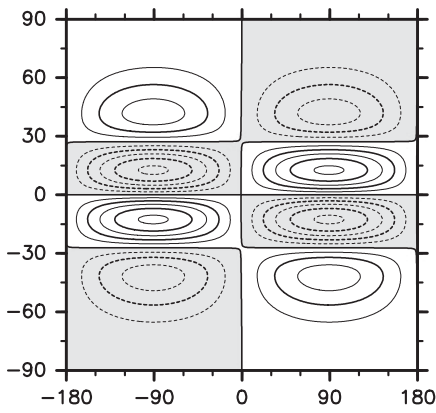

(g) $\omega=-0.42+\mathrm{i}(-0.020)$

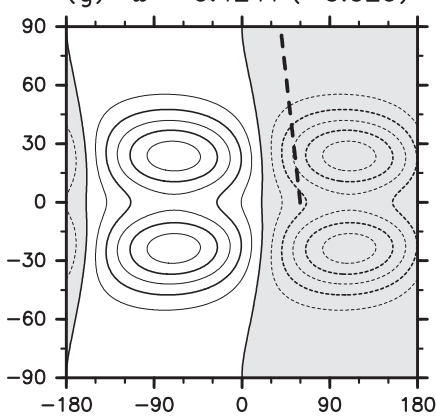

(j) $\omega=-0.26+i(-0.021)$

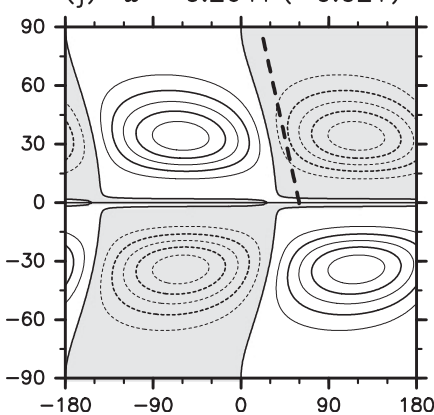

(b) $\quad \omega=-3.40+i(-0.008)$

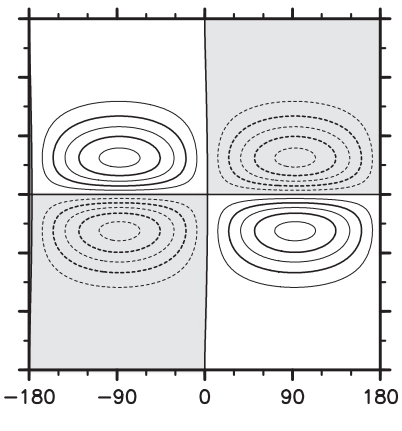

(e) $\omega=11.66+i(-0.011)$

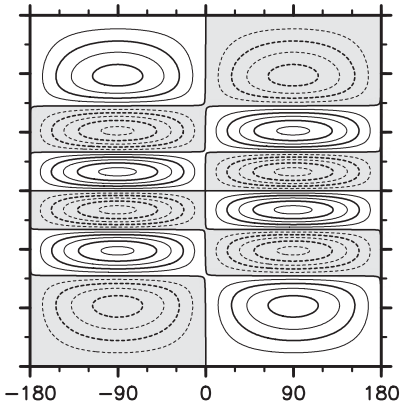

(h) $\quad \omega=-0.19+\mathrm{i}(-0.021)$

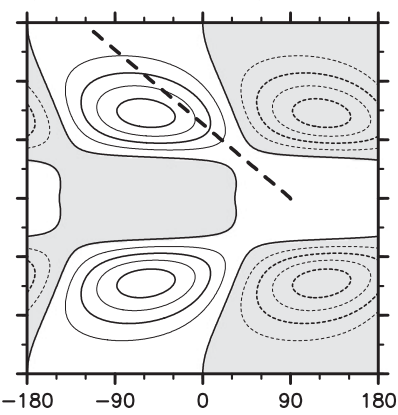

(k) $\quad \omega=-0.15+i(-0.022)$

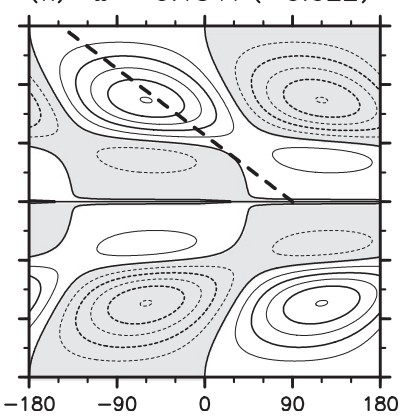

(c) $\omega=4.77+\mathrm{i}(-0.012)$

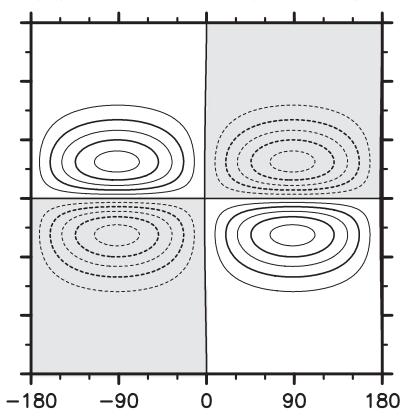

(f) $\omega=13.81+i(-0.012)$

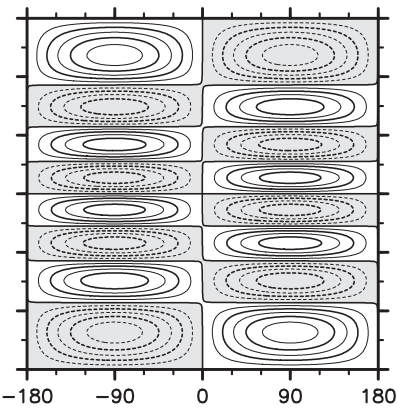

(i) $\quad \omega=-0.13+\mathrm{i}(-0.022)$

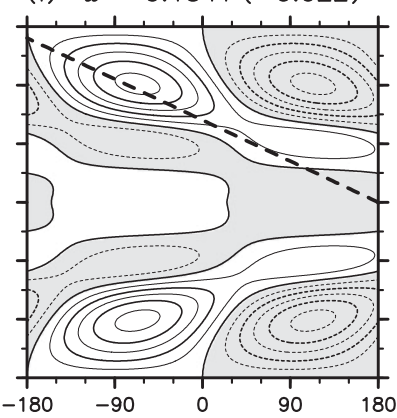

(I) $\omega=-0.11+i(-0.020)$

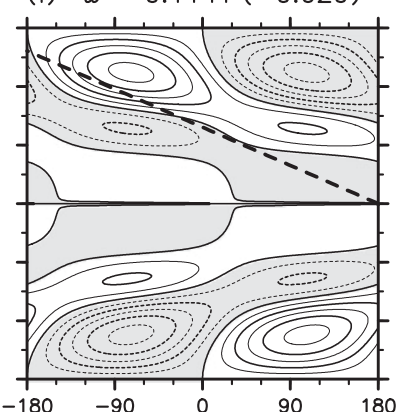

FIG. 2. Geopotential anomaly fields of the distorted Hough modes with the zonal wavenumber $m=1$. (a) Kelvin mode, (b) westward-propagating MRG mode, (c) eastward-propagating MRG mode, (d)-(f) the first three eastward-propagating antisymmetric gravity modes, (g)-(i) the first three symmetric Rossby modes, and (j)-(1) the first three antisymmetric Rossby modes. The frequency of each eigenmode is written above each panel; $i$ is the imaginary unit. Each thick long-dashed line in $(\mathrm{g})-(\mathrm{l})$ has a slope corresponding to the average tilt of the equiphase line of each mode (see text for details). 


\section{a. Description of the weak-nonlinear model}

The weak-nonlinear model introduced here is essentially the same as that used in Kitamura and Ishioka (2007), in which the second-order acceleration induced by waves is evaluated based on a perturbative approach, except that Kitamura and Ishioka (2007) assumed a nonzero basic zonal flow. It is different from other quasi-linear models, such as used in Srinivasan and Young (2012), in that it does not consider the time evolution of the basic zonal flow caused by the second-order acceleration.

First, we expand the dependent variables using a small parameter $\varepsilon$ around a zonally uniform basic flow. That is, the vorticity field $\zeta$, for example, is expanded as

$$
\zeta(\lambda, \mu, t)=\zeta_{0}+\varepsilon \zeta_{1}+\varepsilon^{2} \zeta_{2}+O\left(\varepsilon^{3}\right)
$$

The other dependent variables are expanded similarly. We substitute these expansions into the shallow-water equations [(1)-(3)] and assume that the forcing is of the first order-namely, $F=\varepsilon F_{1}$ - and that the basic state is the state of the rest; that is, $\zeta_{0}=D_{0}=\eta_{0} \equiv 0$ (because we consider a very early stage of the time evolution). Then, we obtain the first-order equations:

$$
\frac{\partial \zeta_{1}}{\partial t}=-\left[\frac{1}{1-\mu^{2}} \frac{\partial}{\partial \lambda}\left(4 \pi \mu U_{1}\right)+\frac{\partial}{\partial \mu}\left(4 \pi \mu V_{1}\right)\right]+d_{\zeta_{1}}+F_{1}
$$

$$
\begin{aligned}
\frac{\partial D_{1}}{\partial t}= & -\left[\frac{1}{1-\mu^{2}} \frac{\partial}{\partial \lambda}\left(4 \pi \mu V_{1}\right)-\frac{\partial}{\partial \mu}\left(4 \pi \mu U_{1}\right)\right] \\
& -\nabla^{2} \eta_{1}+d_{D_{1}}, \quad \text { and }
\end{aligned}
$$

$\frac{\partial \eta_{1}}{\partial t}=-16 \pi^{2} L_{D}^{2} D_{1}+d_{\eta_{1}}$

The zonally averaged second-order equations are obtained as follows:

$$
\begin{aligned}
\frac{\partial \bar{\zeta}_{2}}{\partial t}= & -\frac{\partial}{\partial \mu}\left(4 \pi \mu \bar{V}_{2}\right)+d_{\bar{\zeta}_{2}}-\frac{\partial}{\partial \mu}\left(\overline{\zeta_{1} V_{1}}\right) \\
\frac{\partial \bar{D}_{2}}{\partial t}= & -\frac{\partial}{\partial \mu}\left(4 \pi \mu \bar{U}_{2}\right)-\nabla^{2} \bar{\eta}_{2}+d_{\bar{D}_{2}}-\frac{\partial}{\partial \mu}\left(\overline{\zeta_{1} U_{1}}\right) \\
& -\nabla^{2}\left[\frac{U_{1}^{2}+V_{1}^{2}}{2\left(1-\mu^{2}\right)}\right], \quad \text { and } \\
\frac{\partial \bar{\eta}_{2}}{\partial t}= & -16 \pi^{2} L_{D}^{2} \bar{D}_{2}+d_{\bar{\eta}_{2}}-\frac{\partial}{\partial \mu}\left(\overline{V_{1} \eta_{1}}\right) .
\end{aligned}
$$

At this point, we specify a certain form for neither the forcing term $F_{1}$ nor the dissipation terms $d_{\left(\zeta_{1}, D_{1}, \eta_{1}\right)}$ and $d_{\left.\overline{(}_{2}, \bar{D}_{2}, \bar{\eta}_{2}\right)}$; we only assume that the dissipation terms are linear.

For convenience, we introduce the following vectorform expressions:

$$
\begin{aligned}
& \mathbf{X}=\left(\zeta_{1}, D_{1}, \eta_{1}\right)^{\mathrm{T}}, \\
& \mathbf{Y}=\left(\bar{\zeta}_{2}, \bar{D}_{2}, \bar{\eta}_{2}\right)^{\mathrm{T}}, \\
& \mathbf{F}=\left(F_{1}, 0,0\right)^{\mathrm{T}}, \quad \text { and }
\end{aligned}
$$

$$
\begin{aligned}
\mathbf{A}= & \left(-\frac{\partial}{\partial \mu}\left(\overline{\zeta_{1} V_{1}}\right),-\frac{\partial}{\partial \mu}\left(\overline{\zeta_{1} U_{1}}\right)-\nabla^{2}\left[\overline{\frac{U_{1}^{2}+V_{1}^{2}}{2\left(1-\mu^{2}\right)}}\right],\right. \\
& \left.-\frac{\partial}{\partial \mu}\left(\overline{V_{1} \eta_{1}}\right)\right)^{\mathrm{T}} .
\end{aligned}
$$

In the above expressions, the vector $\left(\zeta_{1}, D_{1}, \eta_{1}\right)^{\mathrm{T}}$, for example, is the abbreviated notation for the vector $\left(\hat{\zeta}_{1}^{1}, \ldots, \hat{\zeta}_{N_{T}}^{N_{T}}, \hat{D}_{1}^{1}, \ldots, \hat{D}_{N_{T}}^{N_{T}}, \hat{\eta}_{1}^{1}, \ldots, \hat{\eta}_{N_{T}}^{N_{T}}\right)^{\mathrm{T}}$, where $\hat{\zeta}_{n}^{m}, \hat{D}_{n}^{m}$, and $\hat{\eta}_{n}^{m}$ are the coefficients in the spherical-harmonics expansion of $\zeta_{1}, D_{1}$, and $\eta_{1}$, respectively.

We can now rewrite the first- and second-order equations in wavenumber space as follows:

$$
\begin{aligned}
& \frac{\partial \mathbf{X}}{\partial t}=\mathbf{L}_{1} \mathbf{X}+\mathbf{F} \quad \text { and } \\
& \frac{\partial \mathbf{Y}}{\partial t}=\mathbf{L}_{2} \mathbf{Y}+\mathbf{A}
\end{aligned}
$$

where $\mathbf{L}_{1}$ and $\mathbf{L}_{2}$ are matrices that correspond to the linear terms (including the dissipation terms) in (10)(12) and (13)-(15), respectively.

Next, we expand the first-order variables by the distorted Hough modes $\mathbf{H}_{l}^{m}$, which are the eigenmodes with the dissipation terms $d_{\left(\zeta_{1}, D_{1}, \eta_{1}\right)}$ taken into account, and the second-order terms by the zonal Hough modes $\mathbf{Z}_{l}$, which are the eigenmodes with the dissipation terms $d_{\left(\bar{\zeta}_{2}, \bar{D}_{2}, \bar{\eta}_{2}\right)}$ taken into account, as follows:

$$
\begin{aligned}
& \mathbf{X}=\sum_{m} \sum_{l} X_{l}^{m} \mathbf{H}_{l}^{m}, \\
& \mathbf{F}=\sum_{m} \sum_{l} F_{l}^{m} \mathbf{H}_{l}^{m}, \\
& \mathbf{Y}=\sum_{l} Y_{l} \mathbf{Z}_{l}, \quad \text { and } \\
& \mathbf{A}=\sum_{l} A_{l} \mathbf{Z}_{l} .
\end{aligned}
$$


Here, $m$ is the zonal wavenumber and $l$ is the index for each mode. The Hough modes above are normalized so that the root-mean-square velocity corresponding to them is unity. Note that since the dissipation terms are included in the eigenvalue problem, we generally obtain the right and left eigenvectors for each eigenvalue. In the above expansion, we use the right eigenvectors. The evolution equations for the expansion coefficients $X_{l}^{m}$ and $Y_{l}^{m}$ become

$$
\begin{aligned}
\frac{d X_{l}^{m}}{d t} & =\sigma_{l}^{m} X_{l}^{m}+F_{l}^{m} \quad \text { and } \\
\frac{d Y_{l}}{d t} & =s_{l} Y_{l}+A_{l} .
\end{aligned}
$$

Here, $\sigma_{l}^{m}$ and $s_{l}$ are the eigenvalues corresponding to $\mathbf{H}_{l}^{m}$ and $\mathbf{Z}_{l}$, respectively. Note that the notation for the eigenvalues of the Hough modes has been changed here $\left(\sigma_{l}^{m}\right.$ corresponds to $-i \omega$ introduced in section 3 ) for convenience.

In the following two subsections, we evaluate the second-order zonal-mean flow acceleration induced by the distorted Hough modes using (26) and (27).

\section{b. Acceleration induced by each Hough mode}

In this subsection, we investigate the second-order acceleration of the zonal-mean flows induced by each Hough mode that is distorted by Newtonian cooling. As stated in section 1 , a similar investigation was conducted for equatorial waves on the $\beta$ plane by Warneford and Dellar (2014, manuscript submitted to J. Fluid Mech.). In the following, we present the results of our analysis, noting similarities to the previous work.

We evaluate the second-order acceleration induced by each distorted Hough mode through the following procedure. To examine only the effect of Newtonian cooling on the Hough modes, we consider no other dissipation processes $\left[d_{\left(\zeta_{1}, D_{1}\right)}=0, d_{\eta_{1}}=-\tau_{\text {rad }}^{-1} \eta_{1}\right]$ and exclude the forcing term $F_{1}$ in the first-order equations and the dissipation term in the second-order equations $\left[d_{\left(\bar{\zeta}_{2}, \bar{D}_{2}, \bar{\eta}_{2}\right)}=0\right]$. Let us consider a situation in which there is only one Hough mode $\mathbf{H}_{l}^{m}$ and its amplitude decays according to (26). The time evolution of its amplitude can be written as

$$
X_{l}^{m}(t)=e^{\sigma_{l}^{m} t}
$$

Here, we assume that the initial value of $X_{l}^{m}(t)$ is unity. Because of the dissipation by Newtonian cooling, the real part of the eigenvalue is negative $\left[\operatorname{Re}\left(\sigma_{l}^{m}\right)<0\right]$ and the amplitude decays with time $\left[X_{l}^{m}(t) \rightarrow 0(t \rightarrow \infty)\right]$. Meanwhile, each of the second-order zonal modes $\mathbf{Z}_{j}$ evolves according to (27). Since A consists of quadratic terms of the first-order variables, the time evolution of $A_{j}$ can be written as

$$
A_{j}(t)=A_{j 0} e^{2 \operatorname{Re}\left(\sigma_{l}^{m}\right) t},
$$

where $A_{j 0}$ is a constant that is determined by (25) and (27). Considering each balanced zonal mode of $\mathbf{Z}_{j}$, the corresponding eigenvalue of which is zero $\left(s_{j}=0\right)$, the time evolution of the amplitude is obtained as the solution of (27) as follows:

$$
Y_{j}(t)=\frac{A_{j 0}}{2 \operatorname{Re}\left(\sigma_{l}^{m}\right)}\left[e^{2 \operatorname{Re}\left(\sigma_{l}^{m}\right) t}-1\right]
$$

Consequently,

$$
Y_{j \infty}=\lim _{t \rightarrow \infty} Y_{j}(t)=-\frac{A_{j 0}}{2 \operatorname{Re}\left(\sigma_{l}^{m}\right)} .
$$

Summing up the contributions from all the balanced zonal modes evaluated in (31) as $Y_{j \infty}$, we can calculate the net zonal acceleration induced by each distorted Hough mode until it decays to zero. Figure 3 shows the result of this calculation for the distorted Hough modes shown in Fig. 2. For the acceleration induced by the Rossby modes, Figs. $3 \mathrm{c}$ and $3 \mathrm{~d}$ in the present study correspond to Fig. 11 in Warneford and Dellar (2014, manuscript submitted to J. Fluid Mech.), except that their analysis is for Rossby waves on an equatorial $\beta$ plane and they also show results with Rayleigh friction. Comparing Figs. 3a-d, it can be clearly seen that the gravity modes produce smaller acceleration and the Rossby modes produce larger acceleration than the other modes. We confirmed that, for the zonal wavenumber $m=1$, higher (i.e., having more nodes in the latitudinal direction) gravity modes that are not shown in Fig. 3 produce even smaller acceleration, and higher Rossby modes produce yet larger acceleration. We also checked that this tendency holds for modes of larger zonal wavenumbers. This tendency can be qualitatively explained by the results in section 3 , because the acceleration of the zonal-mean flow induced by the wave modes is closely related to the tilt of the equiphase lines of those modes. That is, a Hough mode that has a smaller absolute value of the real part of the frequency, as well as an equiphase line that is tilting to a high degree, accelerates the zonalmean flow to a greater extent. Acceleration induced by the distorted Kelvin mode is exceptionally small as seen in Fig. 3a. The reason is likely that the Kelvin mode has no meridional velocity component if it is not distorted by Newtonian cooling. Even when the equiphase line of 
(a)

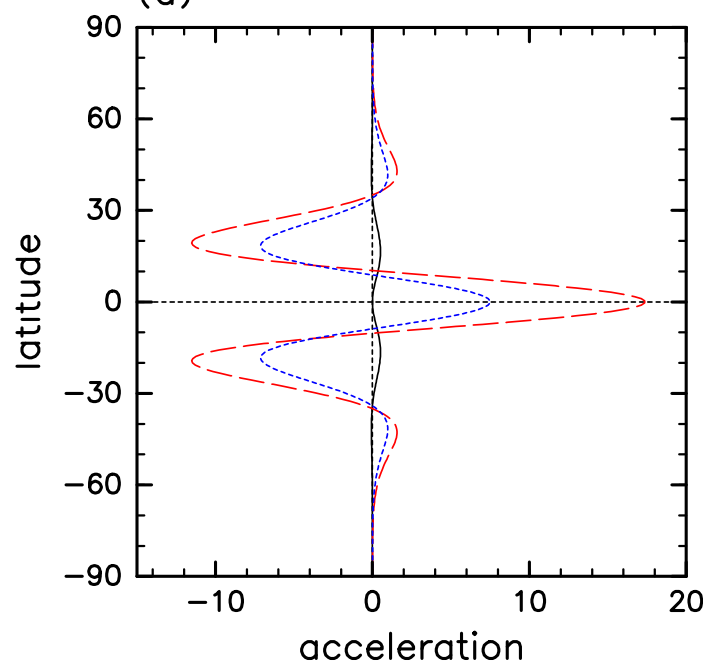

(c)

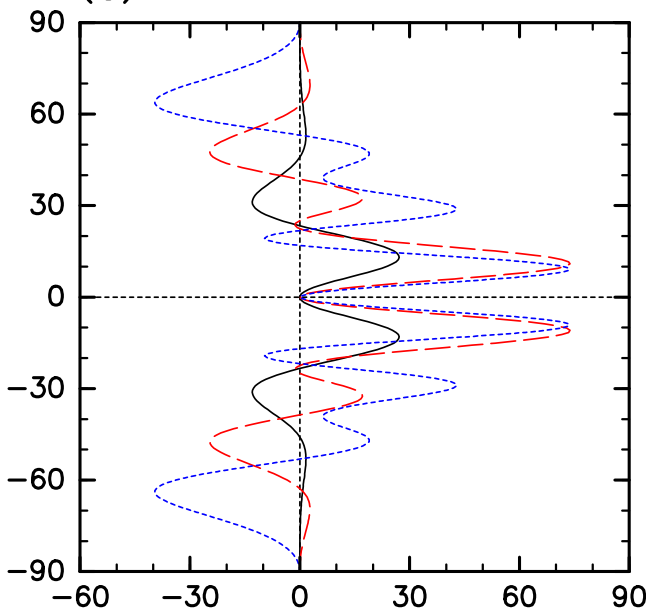

(b)

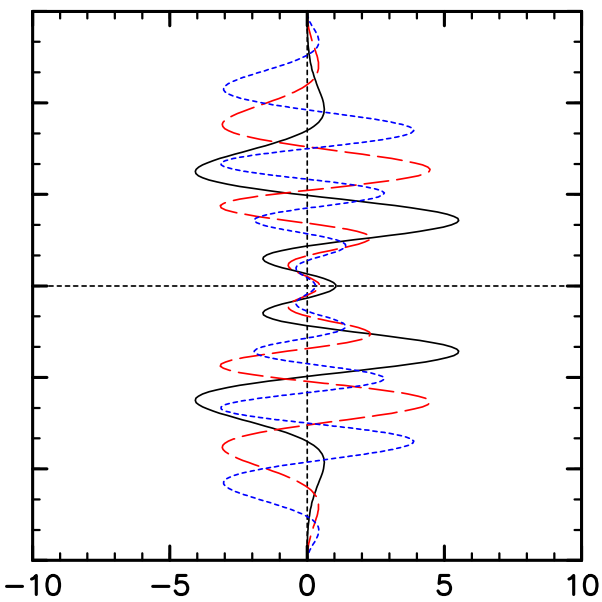

(d)

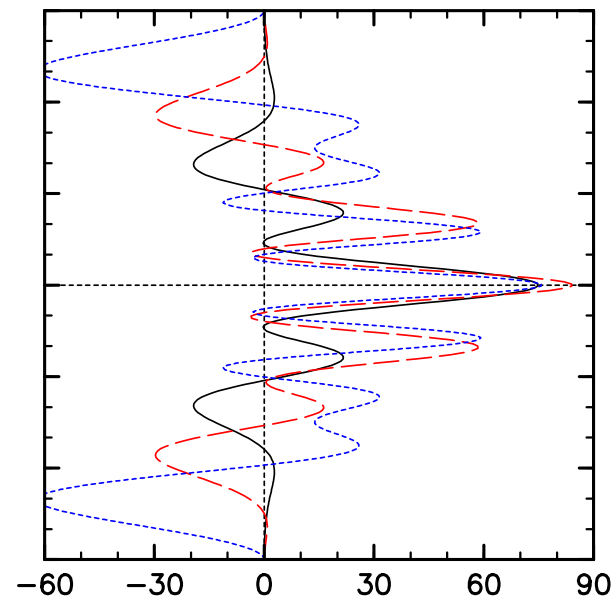

FIG. 3. Net zonal acceleration induced by each distorted Hough mode shown in Fig. 2 until it decays to zero (see text for details of the calculation). (a) Kelvin mode (solid black curve), westward-propagating MRG mode (longdashed red curve), and eastward-propagating MRG mode (short-dashed blue curve); (b) the first three eastwardpropagating antisymmetric gravity modes (solid black curve, long-dashed red curve, short-dashed blue curve, respectively); (c) the first three symmetric Rossby modes (solid black curve, long-dashed red curve, short-dashed blue curve, respectively); and (d) the first three antisymmetric Rossby modes (solid black curve, long-dashed red curve, short-dashed blue curve, respectively).

the Kelvin mode is tilted by Newtonian cooling, the meridional transport of zonal momentum is not increased sufficiently.

As shown in Figs. 3c and 3d, it is remarkable that Rossby modes distorted by Newtonian cooling produce significant prograde acceleration near the equator. Because this acceleration is consistent with the robust formation of the equatorial superrotation shown in the numerical experiments in section 2, it is reasonable to infer that the acceleration induced by the Rossby modes plays a crucial role as argued by Warneford and Dellar (2014, manuscript submitted to J. Fluid Mech.). However, it is a little naive to consider the acceleration due to each mode independently. Since the vector $\mathbf{A}$ consists of quadratic terms of the first-order variables, it also includes contributions from cross terms of different modes. These contributions do not vanish even after averaging over the entire ensemble; therefore, to interpret the results of nonlinear numerical experiments in which many Hough modes are excited, it is insufficient to simply consider the superposition of the accelerations due to each Hough mode. Thus, we have to take into account all Hough modes excited by the stochastic forcing and their correlations, for which the 
quasi-linear-like approach described in the next subsection is suitable.

\section{c. Quasi-linear-like approach}

In this subsection, we investigate the extent to which the acceleration induced by the distorted Hough modes contributes to the emergence of the prograde equatorial jet observed in the numerical experiments in section 2. To this end, we adopt a statistical approach for evaluating the effect of stochastically forced Hough modes and calculate the time evolution of the ensemble-averaged zonal-mean flow. Herein, we refer to this approach as the "quasilinear-like" approach because we follow a very similar line to the method used in the quasi-linear approach adopted by Srinivasan and Young (2012).

\section{1) Evaluation of the ENSEMble-AVERAged SECOND-ORDER ZONAL-MEAN FLOW}

Here, we assume the same type of forcing and dissipation as used in the numerical experiments in section 2 . That is, the dissipation terms are set as follows:

$$
\begin{aligned}
& d_{\left(\zeta_{1}, D_{1}\right)}=-\nu \nabla^{8}\left(\zeta_{1}, D_{1}\right), \quad d_{\eta_{1}}=-\tau_{\mathrm{rad}}^{-1} \eta_{1}-\nu \nabla^{8} \eta_{1}, \\
& d_{\left(\bar{\zeta}_{2}, \bar{D}_{2}\right)}=-\nu \nabla^{8}\left(\bar{\zeta}_{2}, \bar{D}_{2}\right), \quad d_{\bar{\eta}_{2}}=-\tau_{\mathrm{rad}}^{-1} \bar{\eta}_{2}-\nu \nabla^{8} \bar{\eta}_{2},
\end{aligned}
$$

where the values of $\nu$ and $\tau_{\text {rad }}$ are the same as used in section 2 .

To evaluate the covariance of the forcing coefficient $F_{l}^{m}(t)$, we define $\mathbf{G}_{l}^{m}$ as the normalized left eigenvector corresponding to the right eigenvector $\mathbf{H}_{l}^{m}$, so as to satisfy the following biorthogonal condition:

$$
\left(\mathbf{G}_{l_{1}}^{m_{1}}\right)^{\dagger} \mathbf{H}_{l_{2}}^{m_{2}}=\delta_{l_{1} l_{2}} \delta_{m_{1} m_{2}} .
$$

Here, $(\cdot)^{\dagger}$ indicates the complex-conjugate transpose. Then, from (23), we can calculate $F_{l}^{m}(t)$ as

$$
F_{l}^{m}(t)=\mathbf{G}_{l}^{m \dagger} \mathbf{F}(t) .
$$

Since the temporal dependence of the forcing $\mathbf{F}(t)$ is described by the function $\xi_{n}^{m}(t)$ as defined in (5), we can express $\mathbf{F}(t)$ as $\mathbf{F}(t)=\mathbf{R} \Xi(t)$, where $\Xi(t)$ is a column vector consisting of $\xi_{n}^{m}(t)$ and $\mathbf{R}$ is a matrix that consists of $\sqrt{2 n(n+1) \varepsilon_{0} /[(2 n+1) \Delta n]}$ and many zeros. The correlation between $F_{l_{1}}^{m_{1}}(t)$ and $F_{l_{2}}^{m_{2}}\left(t^{\prime}\right)$ can be written as

$$
\begin{aligned}
\left\langle F_{l_{1}}^{m_{1}}(t) F_{l_{2}}^{m_{2}}\left(t^{\prime}\right)^{*}\right\rangle & =\mathbf{G}_{l_{1}}^{m_{1} \dagger} \mathbf{R}\left\langle\Xi(t) \Xi\left(t^{\prime}\right)^{\dagger}\right\rangle \mathbf{R}^{\dagger} \mathbf{G}_{l_{2}}^{m_{2}} \\
& =\mathbf{G}_{l_{1}}^{m_{1} \dagger} \mathbf{R}\left[\boldsymbol{l} \delta\left(t-t^{\prime}\right)\right] \mathbf{R}^{\dagger} \mathbf{G}_{l_{2}}^{m_{2}} \\
& =\mathbf{G}_{l_{1}}^{m_{1} \dagger} \mathbf{R}^{\dagger} \mathbf{G}_{l_{2}}^{m_{2}} \delta\left(t-t^{\prime}\right) \\
& \equiv B_{l_{1} l_{2}}^{m_{1} m_{2}} \delta\left(t-t^{\prime}\right),
\end{aligned}
$$

where $\mathbf{I}$ is the identity matrix and the coefficient $B_{l_{1} l_{2}}^{m_{1} m_{2}}$ is defined as

$$
B_{l_{1} l_{2}}^{m_{1} m_{2}}=\mathbf{G}_{l_{1}}^{m_{1}^{\dagger}} \mathbf{R R}^{\dagger} \mathbf{G}_{l_{2}}^{m_{2}} .
$$

With the random forcing $F_{1}$ described above, (26) forms a Langevin equation. If we assume that the initial value of each $X_{l}^{m}$ is zero, then the time-dependent solution of the covariance of the coefficients of $\mathbf{X}$ becomes

$$
\begin{aligned}
& \left\langle X_{l_{1}}^{m_{1}}(t) X_{l_{2}}^{m_{2}}(t)^{*}\right\rangle \\
& =-\frac{B_{l_{1} l_{2}}^{m_{1} m_{2}}}{\sigma_{l_{1}}^{m_{1}}+\sigma_{l_{2}}^{m_{2}^{*}}}\left\{1-\exp \left[\left(\sigma_{l_{1}}^{m_{1}}+\sigma_{l_{2}}^{m_{2}^{*}}\right) t\right]\right\} .
\end{aligned}
$$

Note that the covariance of $X_{l}^{m}$ is nonzero, so it too must be considered alongside the variance.

Since the vector $\mathbf{A}$ consists of quadratic terms of the first-order variables, each of the expansion coefficients $A_{l}$ can be expressed in a quadratic form of $\mathbf{X}$ as

$$
A_{l}=\mathbf{X}^{\dagger} \mathbf{Q}_{l} \mathbf{X}
$$

where $\mathbf{Q}$ is a matrix that corresponds to the definition of $\mathbf{A}$ in (19). Substituting (22) into the above equation yields

$$
\begin{aligned}
A_{l} & =\left(\sum_{m_{1}} \sum_{l_{1}} X_{l_{1}}^{m_{1}^{*}} \mathbf{H}_{l_{1}}^{m_{1} \dagger}\right) \mathbf{Q}_{l}\left(\sum_{m_{2}} \sum_{l_{2}} X_{l_{2}}^{m_{2}} \mathbf{H}_{l_{2}}^{m_{2}}\right) \\
& =\sum_{m_{1}} \sum_{l_{1}} \sum_{m_{2}} \sum_{l_{2}} X_{l_{1}}^{m_{1}^{*}} X_{l_{2}}^{m_{2}} \mathbf{H}_{l_{1}}^{m_{1} \dagger} \mathbf{Q}_{l} \mathbf{H}_{l_{2}}^{m_{2}} .
\end{aligned}
$$

Since the zonal-mean operation is included in the definition of $\mathbf{A}$, the quadratic form $\mathbf{H}_{l_{1}}^{m_{1} \dagger} \mathbf{Q}_{l} \mathbf{H}_{l_{2}}^{m_{2}}$ can be expressed as

$$
\mathbf{H}_{l_{1}}^{m_{1} \dagger} \mathbf{Q}_{l} \mathbf{H}_{l_{2}}^{m_{2}}=\delta_{m_{1} m_{2}} K_{l l_{1} l_{2}}^{m_{1}},
$$

where we introduce the coefficient $K_{l_{1} l_{2} l_{2}}^{m_{1}}$. Hence, using (34), the ensemble average of $A_{l}$ can be calculated as

$$
\begin{aligned}
\left\langle A_{l}\right\rangle & =\sum_{m} \sum_{l_{1}} \sum_{l_{2}} K_{l_{1} l_{2}}^{m}\left\langle X_{l_{1}}^{m^{*}} X_{l_{2}}^{m}\right\rangle \\
& =-\sum_{m} \sum_{l_{1}} \sum_{l_{2}} \frac{B_{l_{1} l_{2}}^{m m} K_{l_{l_{1} l_{2}}^{m}}^{m}}{\sigma_{l_{1}}^{m}+\sigma_{l_{2}}^{m *}}\left\{1-\exp \left[\left(\sigma_{l_{1}}^{m}+\sigma_{l_{2}}^{m *}\right) t\right]\right\} .
\end{aligned}
$$

Based on (37), the time-dependent solution of the ensemble average of $\left\langle Y_{l}\right\rangle$ in (27) is found to be 


$$
\left\langle Y_{l}\right\rangle=-\sum_{m} \sum_{l_{1}} \sum_{l_{2}} \frac{B_{l_{1} l_{2}}^{m m} K_{l l_{1} l_{2}}^{m}}{\sigma_{l_{1}}^{m}+\sigma_{l_{2}}^{m^{*}}}\left[\frac{1-e^{s_{l} t}}{s_{l}}-\frac{e^{\left(\sigma_{l_{1}}^{m}+\sigma_{l_{2}}^{m *}\right) t}-e^{s_{l} t}}{\sigma_{l_{1}}^{m}+\sigma_{l_{2}}^{m^{*}}-s_{l}}\right] .
$$

Summing up all the contributions from $\left\langle Y_{l}\right\rangle$, we can calculate the time evolution of the ensemble-averaged zonal-mean flow accelerated by the randomly excited distorted Hough modes.

\section{2) COMPARISON WITH THE NONLINEAR TIME EVOLUTIONS}

Now, we compare the theoretical predictions from (34) and (38) with the results of the nonlinear time evolutions shown in section 2. As mentioned in section 2, comparisons are made only for the early stages of the time evolution (i.e., from $t=0$ to $t=1000$ ) to focus on the generation process of the prograde equatorial jet. Limiting the time-evolution period makes it possible to conduct two series of additional numerical experiments beyond those shown in section 2. First, we increase the number of ensemble members from 120 to 400 in order to obtain more accurate statistics. Second, for comparison with the weak-nonlinear model, we conduct numerical experiments with smaller amplitudes of forcing: $\varepsilon_{0}=$ $6.4 \times 10^{-E}$, where $E=9,10$, and 11 . Using the naming convention introduced in section 2 , these experiments are referred to as the "E9," "E10," and "E11" experiments, respectively.

Before focusing on the zonal-mean flow, we examine how well the time evolution of the variance (calculated using 400 ensemble members) of a distorted Hough mode is predicted by the quasi-linear-like approach. Figure 4 shows the time evolutions of the variance of the gravest antisymmetric Rossby mode of $m=42$, which is strongly excited by the forcing, alongside the theoretical predictions from (34) for comparison. To facilitate this comparison, we normalize the variance by the measure of the energy input rate. Consequently, all the theoretical predictions collapse into one curve (Fig. 4, solid black curve). Although the time evolution of the variance in each experiment agrees well with the theoretical prediction at first, it deviates from the prediction after some period of time, which is longer for experiments with smaller forcing amplitudes. The deviation from the theoretical prediction means that the assumptions in the theory are no longer valid: the second-order zonal-mean flow becomes so large that it cannot be considered small relative to the first-order wave components, and the third- and higher-order nonlinear interactions come into play. In fact, we confirmed that the inverse energy

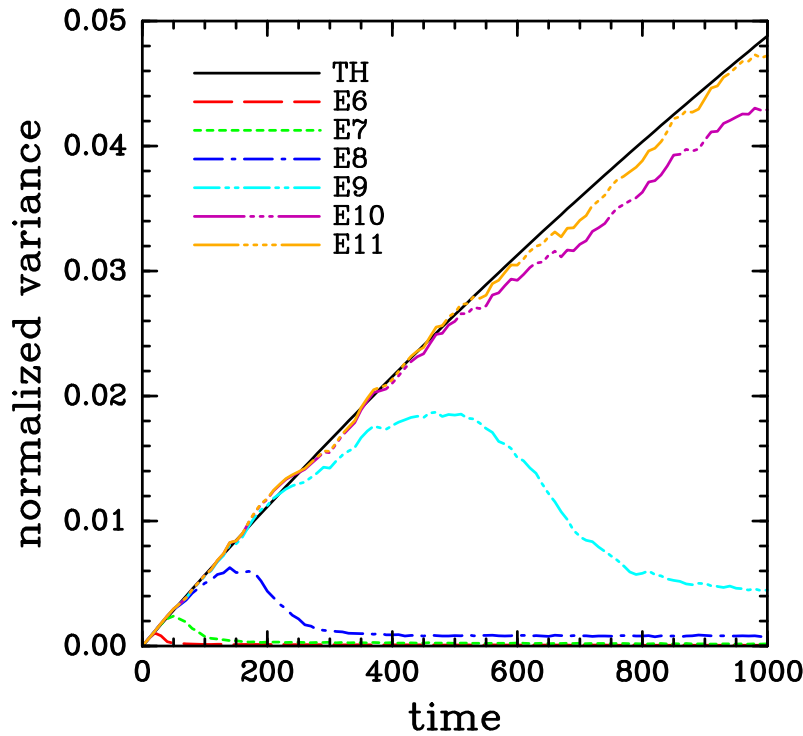

FIG. 4. Time evolution of the variance of the coefficient of the gravest antisymmetric Rossby mode with the zonal wavenumber $m=42$. The solid black curve is the theoretical prediction (see text for details of the calculation). The colored curves are the ensemble averages of 400 nonlinear runs for each experiment: the results for E6-E11 experiments are shown by long-dashed red, short-dashed green, dashed-dotted blue, long-dashed-two-dotted cyan, longdashed-three-dotted purple, and long-dashed-four-dotted yellow curves, respectively. The value of the variance is normalized by the measure of the energy injection rate.

cascade is clearly seen in the energy spectrum before the deviation becomes significant (not shown).

Now we focus on the time evolution of the zonal-mean flow. We check the theoretical prediction of the time evolution [(38)] before comparing it with the result of the nonlinear time evolutions. Dashed red curves in Fig. $5 \mathrm{c}$ show the theoretically predicted time evolution of the zonal-mean flow. Here, the measure of the energy input rate is set to the value used in the E8 experiments. As time goes on, a prograde equatorial jet emerges in the equatorial region $\left(|\varphi| \lesssim 20^{\circ}\right)$ and some wavy patterns appear at high latitudes $\left(|\varphi| \geqslant 80^{\circ}\right)$. At midlatitudes, however, no significant acceleration is observed. We also calculated the same time evolution of the zonalmean flow based on the quasi-linear-like approach, except that only the contribution from the Rossby modes to the term $\left\langle A_{l}\right\rangle$ was included in (37). We obtained almost the same results as dashed red curves in Fig. $5 c$; the difference cannot be seen because of the resolution of the figure. This agreement means that the zonal-mean flow acceleration induced by the Rossby modes is predominant in the weak-nonlinear model. The importance of the Rossby modes for the emergence of the prograde equatorial jet was also discussed by Warneford and Dellar (2014, manuscript submitted to J. Fluid Mech.). 

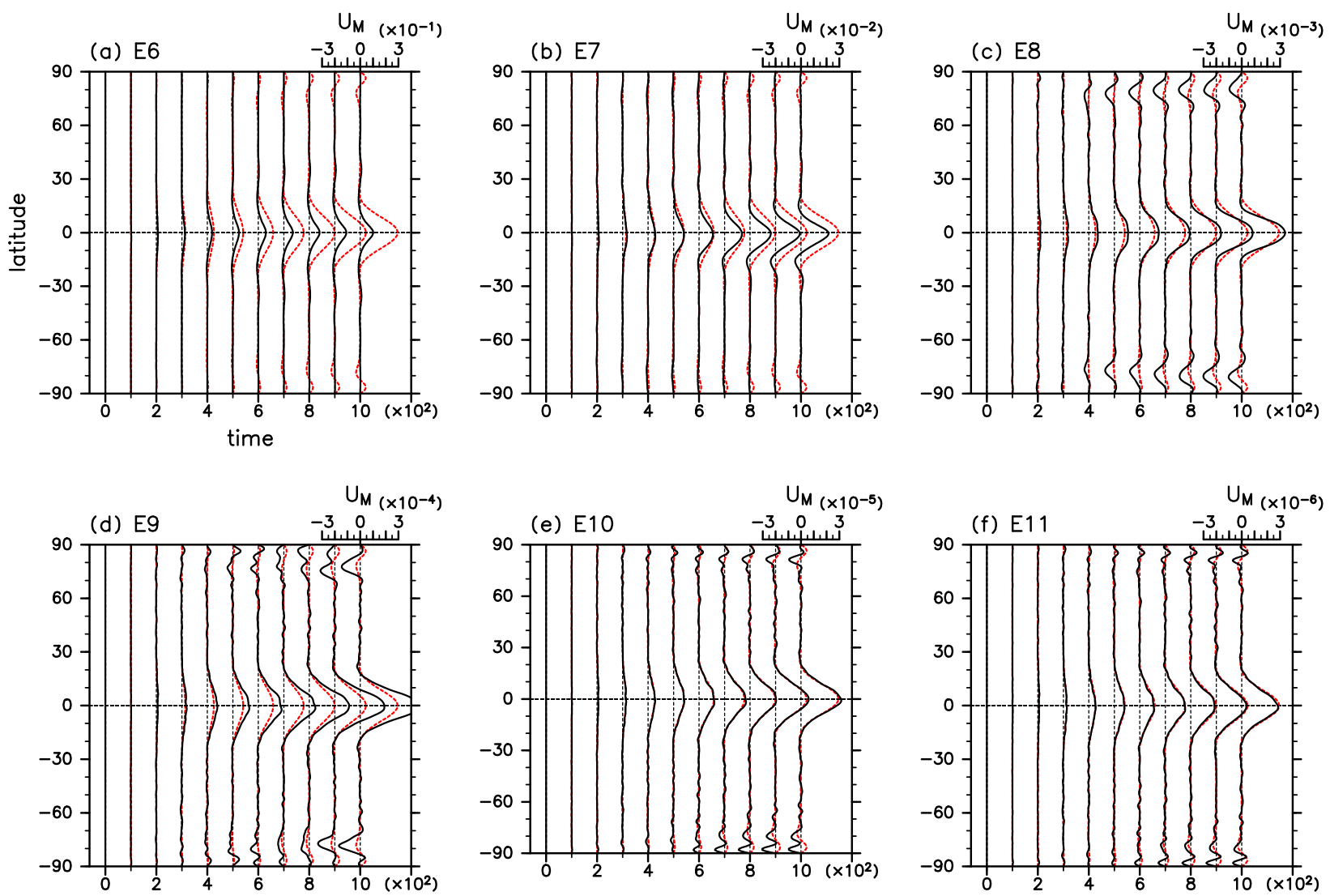

FIG. 5. Dashed red curves indicate snapshots of the ensemble-averaged zonal-mean zonal velocity calculated from the theoretical prediction [(38)] at $t=n \Delta t(n=0,1, \ldots, 10 ; \Delta t=100)$. Solid black curves indicate profiles of the ensemble-averaged zonal-mean zonal velocity for 400 nonlinear runs. (a)-(f) E6-E11 experiments, respectively. The scale for velocity is indicated by a ruler at the upper right of each panel. The dashed vertical lines indicate zero velocity at each time.

Figure 5 compares the time evolution of the zonal-mean flow predicted theoretically in (38) with that of the ensemble-averaged zonal-mean flow obtained in the numerical experiments. For all cases (E6-E11 experiments as shown in Figs. 5a-f, respectively), the time evolutions of the profiles of the zonal-mean flow in the numerical experiments agree qualitatively with the theoretical predictions, in that there appears a prograde equatorial jet, whereas no significant jet is seen at midlatitudes. Moreover, in the early stages of the time evolution, the profiles of the prograde equatorial jet are in good quantitative agreement for E7-E9 experiments as shown in Figs. 5b-d. For E10 and E11, the theoretical and ensemble-averaged results are almost indentical, as shown in Figs. 5e and 5f. To facilitate a quantitative comparison, the time evolutions of the eastward velocity of the ensemble-averaged zonal-mean flow at the equator for E6-E11 experiments are shown in Fig. 6 and the value of the velocity is normalized by the measure of the energy injection rate. As can be seen, the time evolutions of all but that for E6 experiments overlap quite well with the theoretical prediction (Fig. 6, solid black curve) in the early stages. Note that this good agreement following the normalization by $\varepsilon_{0}$ makes clear the importance of the second-order acceleration terms for the formation of the equatorial prograde jet. For E6 experiments, however, the time evolution deviates from the theoretical prediction at around $t \sim 10$, which is soon after the beginning of the simulation. The reason seems to be that the forcing is so strong for E6 experiments that the validity of the weak-nonlinear theory is lost in the initial stages of the time evolution.

It may seem strange that the result of E8 experiments shows better agreement with the theoretical prediction than that of E9 experiments, despite the larger forcing amplitude in E8 experiments (cf. Fig. 5c with Fig. 5d and the dashed-dotted blue curve in Fig. 6a with the longdashed-two-dotted cyan curve in Fig. 6b). By making a quasi-linear model and conducting a similar ensemble experiment to that conducted in this section, we found that this seemingly good agreement with the theory after $t=200$ for E8 experiments is just a coincidence, which is 
$\left(\times 10^{4}\right)(a)$

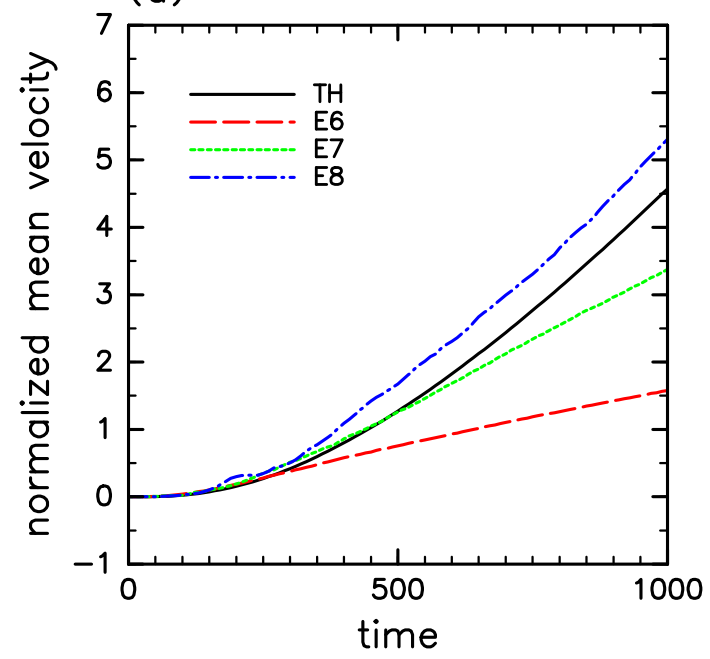

(b)

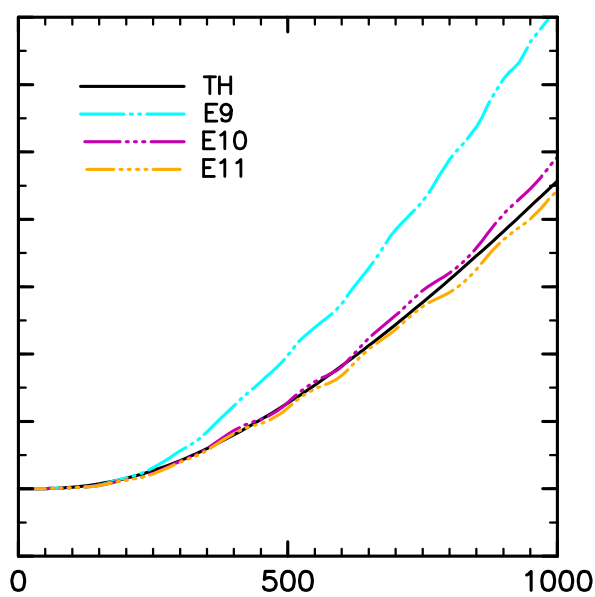

FIG. 6. Time evolution of the ensemble-averaged zonal-mean zonal velocity at the equator. Solid black curves indicate the theoretical prediction (see text for details of the calculation). The colored curves are the ensemble averages of 400 nonlinear runs for each experiment. (a) The results for E6, E7, and E8 experiments are shown by long-dashed red, short-dashed green, and dashed-dotted blue curves, respectively. (b) The results for E9, E10, and E11 experiments are shown by long-dashed-two-dotted cyan, long-dashed-three-dotted purple, and long-dashed-four-dotted yellow curves, respectively. The value of the velocity is normalized by the measure of the energy injection rate.

the result of a combination of effects that are not accounted for in the theory (see appendix for detail).

\section{Summary and discussion}

The main purpose of the present study was to quantitatively determine whether the zonal-mean flow acceleration due to wave modes distorted by Newtonian cooling leads to the formation of the robust equatorial superrotation reported by Scott and Polvani (2008) in forced shallow-water turbulence. For this purpose, we conducted ensemble experiments in section 2 and confirmed the robust formation of equatorial superrotation but also found that some ensemble members formed equatorial subrotation. In section 3, we showed that including a Newtonian cooling term in the shallow-water equations leads to the tilting of the equiphase lines of the Hough modes, and in section 4, following the calculation of the zonal-mean flow acceleration induced by the distorted Hough modes using a weak-nonlinear framework, performed a statistical analysis to evaluate the acceleration induced by the distorted Hough modes that were themselves excited by the stochastic forcing. The evaluated acceleration is almost completely due to Rossby modes, the equiphase lines of which are tilted westward by Newtonian cooling. The acceleration provides a good quantitative explanation for the onset of the equatorial superrotation in the nonlinear time evolutions shown in section 2. Although Yamagata and Philander (1985) and Warneford and Dellar (2014, manuscript submitted to J. Fluid Mech.) dealt with equatorial wave-related subjects similar to those explored in sections 3 and $4 \mathrm{~b}$, respectively, the present study is the first to show quantitatively that the second-order acceleration induced by the distorted Hough modes leads to the onset of equatorial superrotation in a randomly forced shallow-water system with Newtonian cooling.

Since the present paper is motivated by the equatorial superrotation of Jupiter and Saturn, let us compare the equatorial jet speeds for the superrotating runs obtained in section 2 with those of the real planetary atmospheres even though there is a large gap between the model used in the present paper and the real planetary atmospheres. With Jovian parameters (the rotation rate and the radius), the typical value of the nondimensionalized speed of the equatorial jets for the E8 experiments, 0.05, approximately corresponds to $100 \mathrm{~m} \mathrm{~s}^{-1}$ whereas those of the E7 and the E6 experiments, 0.15 and 0.35, approximately correspond to 250 and $580 \mathrm{~m} \mathrm{~s}^{-1}$, respectively, with Saturn parameters. Comparing them with the observed values (e.g., Liu and Schneider 2010), the E8 experiments corresponds to Jupiter whereas the E7 and the E6 experiments correspond to Saturn, roughly speaking.

In the present paper, we focused on the onset of the equatorial superrotation. We believe, however, that the onset is crucial for the subsequent robustness of the equatorial superrotation. Once a sufficiently strong prograde equatorial jet is formed, it can persist owing to either the potential vorticity mixing or the interaction between the zonal flow and waves. Details of these mechanisms are 
discussed in Kitamura and Ishioka (2007). The importance of the initial jet for the location of the subsequent jet sharpening by turbulent mixing is also reported in Dritschel and Scott (2011).

We also note that the initial acceleration mechanism of the prograde equatorial jet discussed in the present study may have some implications on the stochastic structural stability theory (SSST, or S3T) analysis carried out by Farrell and Ioannou (2009) using a quasi-linear model of shallow-water equations on the equatorial $\beta$ plane. Although their S3T analysis demonstrates that both prograde and retrograde equatorial jets are stable and realizable for forcings sufficiently small in amplitude, the nonlinear time evolutions performed in the present paper show the predominance of equatorial superrotation. The initial acceleration of the prograde equatorial jet induced by the wave modes as discussed in the present paper was not taken into account in the S3T analysis, indicating that this is likely the reason for the differences in the findings of the two studies.

As summarized above, we explored the origin of the equatorial superrotation emerging from shallow-water turbulence on a rotating sphere with Newtonian cooling. The robust formation of the superrotation in this system, first reported by Scott and Polvani (2008), was confirmed in section 2. However, there is a small but notable difference between their results and ours. Whereas they obtained a prograde equatorial jet in all cases with Newtonian cooling as the sole dissipation process at large scales, we obtained an equatorial retrograde jet for some ensemble members. As mentioned in section 2, there are two differences in the experimental setup: one is the hyperviscosity coefficient (corresponding to the difference in the truncation wavenumber) and the other is the time-integration scheme. If the value of $L_{D}$ had been smaller, the hyperviscosity term might have changed the experimental result because its dissipation effect could also have distorted the Hough modes and affected the second-order acceleration as a result. For this reason, we set $L_{D}=0.1$ in section 2 . The ratio of the damping rate caused by the Newtonian cooling to that due to the hyperviscosity is $\tau_{\text {rad }}^{-1} /\left\{\nu[n(n+1)]^{4}\right\} \approx 186$ for $n=44$ (the highest total wavenumber at which the forcing is imposed), which is still sufficiently larger than unity. This is not the case for setting $L_{D}=0.025$, in which the ratio becomes about 12 . In addition, in order to check whether the emergence of the equatorial subrotation in section 2 is due to the hyperviscosity (and the spatial resolution of the model), we conducted additional experiments where the resolution is increased to $N_{T}=341$ and the hyperviscosity coefficient $\nu$ is decreased in accordance with the definition in section 2 . Because these experiments are quite time consuming, we only managed to conduct 20 ensemble experiments each for the E6 and E7 settings. Among the 20 members, 16 members yielded a prograde equatorial jet and the other members yielded a retrograde equatorial jet for E6, whereas all of the members yielded a prograde equatorial jet for E7. Because a retrograde equatorial jet emerges even for these higher-resolution and smallerhyperviscosity experiments, it is suggested that the equatorial subrotation obtained in section 2 is not a spurious result caused by the low resolution or the hyperviscosity. On the other hand, it is possible that the time-integration schemes caused the difference in the experimental results. Whereas we used the classical fourth-order Runge-Kutta scheme, Scott and Polvani (2008) used the second-order leapfrog scheme with a Robert-Asselin time filter. This time filter tends to preferentially damp high-frequency oscillations, such as high-order gravity modes. Since the acceleration mechanism proposed in the present study originates mainly from Rossby modes, which have lower frequencies, the preferential damping of high-frequency modes may have exaggerated the frequency of emergence of prograde equatorial jets in their study. In fact, Kitamura and Ishioka (2007) reported that the difference in timeintegration schemes can lead to differences in experimental results for a shallow-water system.

Since the equatorial subrotation obtained in section 2 does not seem to be a spurious result as discussed above, let us consider the reason why the frequency of the emergence of prograde equatorial jets depends on the forcing amplitude. In the ensemble experiments in section 2, all of the ensemble members exhibit a prograde equatorial jet for the E8 experiments and for the experiments with smaller forcing amplitudes, whereas at least $10 \%$ of the members exhibit a retrograde equatorial jet for the E6 and E7 experiments. Based on the fact that the time evolution of the variance of a Hough mode deviates from the theoretical prediction sooner for the E6 and E7 experiments than for the E8-E11 experiments as shown in Fig. 4, a possible explanation for this dependence on the forcing is that the energy injection rate is too large for the acceleration mechanism described in section 4 to work and produce a sufficiently strong prograde equatorial jet in the initial stages of the time evolution for the E6 and E7 experiments. With the prograde acceleration not lasting long enough in the E6 and E7 experiments, equatorial subrotation resulting from the random vorticity field in the initial stages can survive and be reinforced in some cases by subsequent wave-mean flow interactions as discussed in Kitamura and Ishioka (2007).

To explore the dependence of the frequency of the emergence of prograde equatorial jets on the energy injection rate further, we conducted additional ensemble experiments with a greater energy injection rate. The 
settings are as described in section 2 except that the measure of the energy injection rate is set to $\varepsilon_{0}=6.4 \times$ $10^{-5}$. Among the 120 members in these E5 experiments, only three members exhibit a retrograde equatorial jet, whereas 117 members exhibit a prograde equatorial jet (not shown). Also taking into account the fact that the number of the members with equatorial superrotation in the E6 experiments, 105, is slightly larger than that of the E7 experiments, 99, as shown in section 2, the frequency of the emergence of prograde equatorial jets increases as the forcing amplitude increases among the E5, E6, and E7 experiments. Based on the discussion in the previous paragraph, it is unlikely that the acceleration mechanism described in section 4 alone plays a major role in the emergence of these prograde equatorial jets. Instead, there must be another mechanism that can work effectively in the cases of larger forcing amplitudes to produce the prograde equatorial jets. The S3T theory proposed by Farrell and Ioannou (2009) provides a possible mechanism in which it is demonstrated that only prograde equatorial jets are stable for sufficiently large forcing amplitudes. Although it may be interesting to explore this possibility, quantitative comparison between their theory and our results is difficult because of the differences in the governing equations and the experimental parameters adopted in the present study and in Farrell and Ioannou (2009). The exploration may be a good subject for our future work.

It is finally necessary to discuss the reason why the Newtonian cooling term causes the equiphase line of the Rossby modes to tilt westward, because this phenomenon is crucial for the formation of the equatorial superrotation. The fundamental dynamics of a Rossby wave can be described by the quasigeostrophic potential vorticity (QGPV) equation, which is derived from the shallow-water equations [(1)-(3)] when both the Rossby and Froude numbers are sufficiently small. As discussed by Scott and Polvani (2007) and later by Warneford and Dellar (2014, manuscript submitted to J. Fluid Mech.), Newtonian cooling is expressed in the QGPV equation as a term with a dissipation coefficient that is proportional to $\mu^{2}$. Thus, the dissipation rate is larger at higher latitudes and almost zero near the equator. With such a latitude-dependent dissipation, each eigenmode has to be accompanied by an energy transfer from lower latitudes to higher latitudes in order to maintain its structure. This poleward energy transfer indicates a poleward group velocity, which manifests itself as the westward tilt of the equiphase lines with increasing absolute value of latitude for Rossby modes. To summarize, the essential cause of the prograde acceleration at the equator in a shallow-water system with Newtonian cooling is the latitudinal dependence of the dissipation acting on the Rossby modes. This implies that equatorial superrotation can emerge similarly in other systems through acceleration due to Rossby modes if the systems have latitudinally dependent dissipation processes. For example, Schneider and Liu (2009) and Liu and Schneider (2010) conducted three-dimensional general circulation model (GCM) experiments intending to simulate the Jovian atmosphere and obtained a strong equatorial superrotation, which was quantitatively similar to that actually observed on Jupiter. Schneider and Liu (2009; see their appendix B, section h) obtained such a strong equatorial superrotation only by considering latitudinally dependent Rayleigh friction, which was introduced as a simplified model of the magnetohydrodynamics drag effect in the Jovian atmosphere. Hence, it is very likely that a similar acceleration mechanism to that shown in the present study contributes, at least partly, to the formation of the strong equatorial superrotation obtained in their three-dimensional GCM experiments.

Acknowledgments. We are grateful to Drs. R. K. Scott, T. J. Dunkerton, J. Y.-K. Cho, M. E. McIntyre, Y.-Y. Hayashi, M. Yamada, S. Yoden, and S. Takehiro for fruitful discussions and instructive comments. We also thank two anonymous reviewers for useful comments and suggestions. The ISPACK numerical library (http://www.gfd-dennou. org/arch/ispack/) was used for the computations and the GFD-DENNOU library (http://www.gfd-dennou.org/arch/ $\mathrm{dcl} /$ ) was used to draw the figures. This research was supported in part by the National Science Foundation under Grant NSF PHY11-25915. Izumi Saito is supported by Grant-in-Aid for JSPS Fellows.

\section{APPENDIX}

\section{The Quasi-Linear Experiment}

The quasi-linear approximation to (1)-(3) is obtained here. We express each of the dependent variables as the sum of its zonal-mean component and the deviation from it as follows:

$$
\zeta=\bar{\zeta}+\zeta^{\prime}, \quad D=\bar{D}+D^{\prime}, \quad \eta=\bar{\eta}+\eta^{\prime}
$$

and substitute (A1) into (1)-(3). Taking the zonal means yields

$$
\begin{aligned}
\frac{\partial \bar{\zeta}}{\partial t}= & -\frac{\partial}{\partial \mu}[(4 \pi \mu+\bar{\zeta}) \bar{V}]+d_{\bar{\zeta}}-\frac{\partial}{\partial \mu}\left(\overline{\zeta^{\prime} V^{\prime}}\right), \\
\frac{\partial \bar{D}}{\partial t}= & -\frac{\partial}{\partial \mu}[(4 \pi \mu+\bar{\zeta}) \bar{U}]+d_{\bar{D}}-\frac{\partial}{\partial \mu}\left(\overline{\zeta^{\prime} U^{\prime}}\right) \\
& -\nabla^{2}\left[\bar{\eta}+\frac{\bar{U}^{2}+\bar{V}^{2}}{2\left(1-\mu^{2}\right)}+\frac{\overline{U^{\prime 2}+V^{\prime 2}}}{2\left(1-\mu^{2}\right)}\right], \text { and }
\end{aligned}
$$




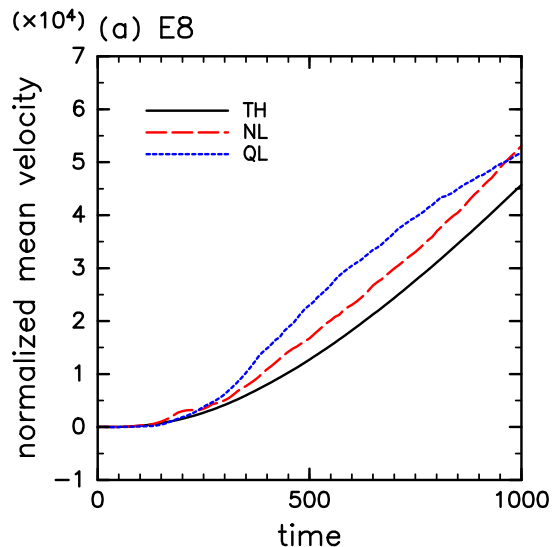

(b) E9

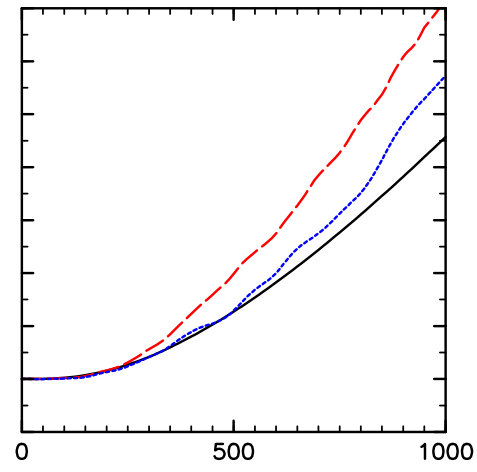

(c) E10

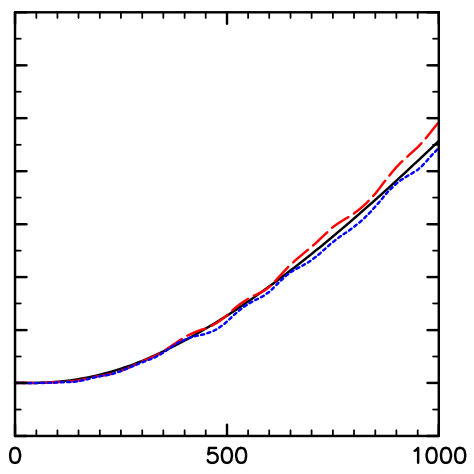

FIG. A1. Time evolution of the ensemble-averaged zonal-mean zonal velocity at the equator for (a) E8, (b) E9, and (c) E10 experiments. Solid black curves indicate the theoretical prediction as in Fig. 6. Long-dashed red and short-dashed blue curves indicate the results of the nonlinear and quasi-linear models, respectively. The value of the velocity is normalized by the measure of the energy injection rate.

$\frac{\partial \bar{\eta}}{\partial t}=-16 \pi^{2} L_{D}^{2} \bar{D}+d_{\bar{\eta}}-\frac{\partial}{\partial \mu}(\bar{V} \bar{\eta})-\frac{\partial}{\partial \mu}\left(\overline{V^{\prime} \eta^{\prime}}\right)$.

Next, subtracting (A2)-(A4) from (1)-(3) and neglecting the second-order terms of the deviations gives

$$
\begin{aligned}
\frac{\partial \zeta^{\prime}}{\partial t}= & -\left\{\frac{1}{1-\mu^{2}} \frac{\partial}{\partial \lambda}\left[(4 \pi \mu+\bar{\zeta}) U^{\prime}+\zeta^{\prime} \bar{U}\right]\right. \\
& \left.+\frac{\partial}{\partial \mu}\left[(4 \pi \mu+\bar{\zeta}) V^{\prime}+\zeta^{\prime} \bar{V}\right]\right\}+d_{\zeta^{\prime}}+F, \\
\frac{\partial D^{\prime}}{\partial t}= & -\left\{\frac{1}{1-\mu^{2}} \frac{\partial}{\partial \lambda}\left[(4 \pi \mu+\bar{\zeta}) V^{\prime}+\zeta^{\prime} \bar{V}\right]\right. \\
& \left.-\frac{\partial}{\partial \mu}\left[(4 \pi \mu+\bar{\zeta}) U^{\prime}+\zeta^{\prime} \bar{U}\right]\right\} \\
& -\nabla^{2}\left(\eta^{\prime}+\frac{U^{\prime} \bar{U}+V^{\prime} \bar{V}}{1-\mu^{2}}\right)+d_{D^{\prime}}, \quad \text { and } \\
\frac{\partial \eta^{\prime}}{\partial t}= & -16 \pi^{2} L_{D}^{2} D^{\prime}+d_{\eta^{\prime}} .
\end{aligned}
$$

Equations (A2)-(A7) form the quasi-linear approximations of the shallow-water equations on a sphere. Unlike the system described by (10)-(15) considered in the main text, here the time evolutions of the deviations are affected by changes in the zonal-mean flow. In both systems, however, the third- and higher-order nonlinear interactions are neglected and hence the inverse energy cascade does not occur. We conduct ensemble experiments using the quasi-linear model described above with 400 ensemble members, employing the same viscosity, parameters, and forcing form as used in section 2 . Figure A1 shows a comparison of the time evolutions of the ensemble-averaged zonal-mean flow at the equator for the full-nonlinear and quasi-linear models with the theoretical predictions for E8, E9, and E10 experiments. The result for E8 experiments (Fig. A1a) shows that after $t=200$, the time evolution of the quasi-linear model deviates from the theory to a greater extent than that of the full-nonlinear model. This implies that, for E8 experiments, the relatively good agreement between the time evolution of the full-nonlinear model and the theory after $t=200$ is just a coincidence, which may be caused by effects that are not considered in the theory. On the other hand, for E9 experiments, the time evolution of the quasi-linear model shows better agreement with the theory than that of the full-nonlinear model (Fig. A1b). For E10 experiments, the time evolutions of both the quasi-linear and full-nonlinear models are in almost complete agreement with the theoretical prediction (Fig. A1c). These two results for E9 and E10 experiments indicate that the level of agreement obtained between the time evolutions of the full-nonlinear model and the theory is not just a coincidence.

\section{REFERENCES}

Andrews, D. G., and M. E. McIntyre, 1976: Planetary waves in horizontal and vertical shear: The generalized Eliassen-Palm relation and the mean zonal acceleration. J. Atmos. Sci., 33, 2031-2048, doi:10.1175/1520-0469(1976)033<2031:PWIHAV>2.0.CO;2.

Busse, F. H., 1983: Convection-driven zonal flows in the major planets. Pure Appl. Geophys., 121, 375-390, doi:10.1007/BF02590147.

Cho, J. Y.-K., and L. M. Polvani, 1996: The emergence of jets and vortices in freely evolving, shallow-water turbulence on a sphere. Phys. Fluids, 8, 1531-1552, doi:10.1063/1.868929.

Dritschel, D. G., and M. E. McIntyre, 2008: Multiple jets as PV staircases: The Phillips effect and the resilience of eddytransport barriers. J. Atmos. Sci., 65, 855-874, doi:10.1175/ 2007JAS2227.1.

— and R. K. Scott, 2011: Jet sharpening by turbulent mixing. Philos. Trans. Roy. Soc. London, A369, 754-770, doi:10.1098/ rsta.2010.0306. 
Farrell, B. F., and P. J. Ioannou, 2009: Emergence of jets from turbulence in the shallow-water equations on an equatorial beta plane. J. Atmos. Sci., 66, 3197-3207, doi:10.1175/2009JAS2941.1.

Hayashi, Y.-Y., S. Nishizawa, S. Takehiro, M. Yamada, K. Ishioka, and S. Yoden, 2007: Rossby waves and jets in two-dimensional decaying turbulence on a rotating sphere. J. Atmos. Sci., 64, 4246-4269, doi:10.1175/2007JAS2209.1.

Kitamura, Y., and K. Ishioka, 2007: Equatorial jets in decaying shallow-water turbulence on a rotating sphere. J. Atmos. Sci., 64, 3340-3353, doi:10.1175/JAS4015.1.

Liu, J., and T. Schneider, 2010: Mechanisms of jet formation on the giant planets. J. Atmos. Sci., 67, 3652-3672, doi:10.1175/2010JAS3492.1.

Longuet-Higgins, M. S., 1968: The eigenfunctions of Laplace's tidal equations over a sphere. Philos. Trans. Roy. Soc. London, A262, 511-607, doi:10.1098/rsta.1968.0003.

Matsuno, T., 1966: Quasi-geostrophic motions in the equatorial area. J. Meteor. Soc. Japan, 44, 25-43.

Nozawa, T., and S. Yoden, 1997: Formation of zonal band structure in forced two-dimensional turbulence on a rotating sphere. Phys. Fluids, 9, 2081-2093, doi:10.1063/1.869327.

Okuno, A., and A. Masuda, 2003: Effect of horizontal divergence on the geostrophic turbulence on a beta-plane: Suppression of the Rhines effect. Phys. Fluids, 15, 56-65, doi:10.1063/1.1524188.

Rhines, P. B., 1975: Waves and turbulence on a beta-plane. J. Fluid Mech., 69, 417-443, doi:10.1017/S0022112075001504.

Schneider, T., and J. Liu, 2009: Formation of jets and equatorial superrotation on Jupiter. J. Atmos. Sci., 66, 579-601, doi:10.1175/ 2008JAS2798.1.
Scott, R. K., and L. M. Polvani, 2007: Forced-dissipative shallowwater turbulence on the sphere and the atmospheric circulation of the giant planets. J. Atmos. Sci., 64, 3158-3176, doi:10.1175/ JAS4003.1.

$\longrightarrow$, and - 2008: Equatorial superrotation in shallow atmospheres. Geophys. Res. Lett., 35, L24202, doi:10.1029/ 2008GL036060.

Srinivasan, K., and W. R. Young, 2012: Zonostrophic instability. J. Atmos. Sci., 69, 1633-1656, doi:10.1175/JAS-D-11-0200.1.

Theiss, J., 2004: Equatorward energy cascade, critical latitude, and the predominance of cyclonic vortices in geostrophic turbulence. J. Phys. Oceanogr., 34, 1663-1678, doi:10.1175/ 1520-0485(2004)034<1663:EECCLA>2.0.CO;2.

Vallis, G. K., and M. E. Maltrud, 1993: Generation of mean flows and jets on a beta plane and over topography. J. Phys. Oceanogr., 23, 1346-1362, doi:10.1175/1520-0485(1993)023<1346: GOMFAJ $>2.0 . \mathrm{CO} ; 2$.

Williams, G. P., 1978: Planetary circulations: 1. Barotropic representation of Jovian and terrestrial turbulence. J. Atmos. Sci., 35, 1399-1426, doi:10.1175/1520-0469(1978)035<1399: PCBROJ $>2.0$.CO;2.

Yamagata, T., and S. G. H. Philander, 1985: The role of damped equatorial waves in the oceanic response to winds. J. Oceanogr. Soc. Japan, 41, 345-357, doi:10.1007/BF02109241.

Yoden, S., and M. Yamada, 1993: A numerical experiment on twodimensional decaying turbulence on a rotating sphere. J. Atmos. Sci., 50, 631-644, doi:10.1175/1520-0469(1993)050<0631: ANEOTD $>2.0 . \mathrm{CO} ; 2$. 